\title{
ON-OFF ITERATIVE ADAPTIVE CONTROLLER FOR LOW-POWER MICRO-ROBOTIC STEP REGULATION
}

\author{
Bongsu Hahn and Kenn Oldham
}

\begin{abstract}
A novel model-free iterative adaptive controller is presented for lowpower control of piezoelectric actuators. The controller uses simple adaptation rules based on known general behavior of piezoelectric actuators to adjust onoff switching times to drive piezoelectric actuators through a desired transient step motion. Adaptation rules are based on small numbers of measurements taken during each iteration of the actuator movement. Combined with the use of only on-off control inputs, controller implementation can be possible at much lower overall power levels than would be needed to implement a conventional control strategy such as through pulse-width-modulation (PWM) with real-time feedback. Such power savings are particularly important for the intended controller application to piezoelectric microactuators driving autonomous terrestrial micro-robots. A method for predicting convergence of systems with nominally linear dynamics and unknown, bounded nonlinearities is described, and applied to a sample target piezoelectric actuator. The controller is tested in simulation and experimentally on a piezoelectric cantilever actuator, and shows predicted convergence to the desired response.
\end{abstract}

Key Words: Adaptive control, iterative learning control, MEMS.

\section{INTRODUCTION}

In this paper, we present a model-free, iterativelyadaptive, on-off controller for regulating the motion of piezoelectric actuators intended for terrestrial microrobotic locomotion. A critical challenge for servocontrol of autonomous micro-systems is to minimize power consumption, due to the extremely small mass or size permitted for the power system. These power constraints will be especially strict for autonomous

Manuscript received January 31, 2010; revised July 21, 2010; accepted December 28, 2010.

Bongsu Hahn and Kenn Oldham (corresponding author) are with the Department of Mechanical Engineering, University of Michigan, Ann Arbor, MI 48109, U.S.A. (e-mail: suhahn@umich.edu; oldham@umich.edu).

This research was supported in part by grant HR001-081-0040 from the U.S. Defense Advanced Research Projects Ageny and grant 0954422 from the National Science Foundation. terrestrial micro-robots, such as that shown in the concept drawing in Fig. 1(a).

Such robots would have dimensions on the order of a few millimeters to a centimeter, and payload capacities of a few to a few hundred milligrams, based on structural analysis of microelectromechanical system (MEMS) actuator capabilities and structural properties of MEMS flexural joints [2]. Such a small payload translates into only about $100 \mu \mathrm{W}$ to $1 \mathrm{~mW}$ of power available to control motion of a given leg, requiring control strategies that aggressively limit power to actuators, interface circuitry, and sensors.

The first novel element of our strategy for performing extremely low-power control of microrobotic actuators is to rely on on-off switching control. On-off controllers are often used by engineering systems with only basic actuator capabilities, limited computation resources, and/or tight energy constraints. In the case of autonomous micro-robotics, thinfilm piezoelectric micro-actuators are a particularly promising actuator type, with potential to provide much 


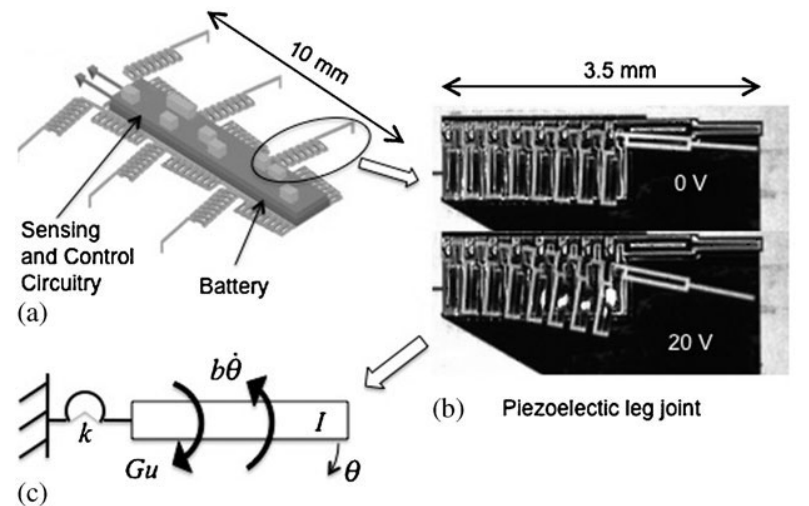

Fig. 1. (a) Concept drawing of a millimeter-scale miro-robot driven by piezoelectric microactuators; b) Prototype thin film piezoelectric leg joint; and (c) Lumped parameter model (Courtesy U.S. Army Research Lab. [1]).

larger micro-robotic ranges of motion and locomotion speeds than those of previous micro-robots based on electrostatic or thermal microactuators. A sample prototype micro-robotic leg joint based on thin-film piezoelectric actuation is shown in Fig. 1(b) (courtesy U.S. Army Research Laboratory). In terms of power consumption, piezoelectric actuators appear as capacitive loads, but require relatively high voltages to operate $(20$ to $30 \mathrm{~V})$. This results in both conventional analog and pulse-width modulation (PWM) controllers being inefficient. Under analog control, for example, typically $95 \%$ of all servo system power usage for piezoelectric actuators is wasted in amplifiers [3]. While this usually drives system designers to PWM techniques, even these approaches may require excessive power, due to the repeated charging and discharging of the capacitive load necessary at high-frequency.

On-off control, in which only a few switching transitions between 'on' and 'off' states are used to guide actuator motion, can dramatically reduce power consumption of piezoelectric actuators by reducing the number of times that the actuator is charged. In return, on-off control entails a degradation of system performance, due to oscillations between switches, excitation of high-frequency dynamics, and dependence of response time on open-loop properties. Nonetheless, under extreme power constraints, such as those on an autonomous micro-robot, this trade-off may be acceptable. However, an additional obstacle to on-off control implementation is that most existing on-off control algorithms rely on accurate knowledge of system dynamics [4-6], for open-loop situations, or comparatively high-frequency sensing in order to implement decision rules on feedback measurements [7]. Thinfilm piezoelectric actuators, while having smaller hysteresis than most piezoelectric actuators, display both hysteresis and nonlinear gains that are difficult to predict or model, as is micro-scale damping behavior.

The second innovation presented here is to utilize step-to-step, or iterative, adaptations of the on-off switching sequence to adjust for unknown dynamics with relatively low sensor sampling rates. Power consumption of sensing transducers and circuitry used in MEMS devices, such as capacitive position sensors, increases greatly with sampling rate. This makes it very difficult to implement real-time feedback control or model adaptation within strict power budgets. Fortunately, in the case of terrestrial micro-robotics, actuator motions are expected to repeat many times, as in walking or running gaits. This allows for lower sampling frequency measurements, compared over several iterations of a given movement, to guide on-off switching sequences producing a desired displacement or trajectory. For certain classes of system, this can be performed without any model of the system in the controller itself, which further simplifies computational requirements on a micro-robot.

The on-off iterative adaptive scheme to be described here is targeted for control of micro-robotic steps, by directly adapting specific time periods within an on-off sequence using measurements taken over successive steps, without any model of system dynamics. This requires limiting the desired motion to specific profiles (in this case, smooth steps to various reference levels) and only transient performance may be predicted reliably, but the benefit is an extremely simple control law to implement, with guarantees on convergence for systems with bounded nonlinearity. Our approach differs from previous adaptive on-off controllers, which use model-based adaptation schemes, where the form of the system dynamics is known but not all of the parameters $[8,9]$. Alternate, related adaptive controllers have utilized fuzzy logic or sliding mode control, but to date have only been used to adapt a single control parameter [10-12]. In contrast, there has been significant work on model-free control of unknown, nonlinear systems, but this has not been applied to on-off control $[13,14]$.

To summarize the contribution of this paper, then, we present a novel on-off, model-free adaptive controller for low-power control of micro-robotic leg joints. This provides a unique perspective on managing energy losses in feedback control of microactuators, and some basic analyitical techniques for examining behavior of the resulting system. Following this introduction, we describe the system to be controlled and the procedure for use of our model-free adaptive on-off controller algorithm. We then describe a method of 
convergence analysis, and examine a specific controller for repeated stepping motions of a 2nd-order system, proving convergence of that controller to desired motions under certain conditions. We present simulation and preliminary experimental results, and conclude with a discussion of the benefits of our analysis and implications for more general problems.

\section{SYSTEM DEFINITION}

\subsection{System dynamics, input, and conditions}

The micro-robotic appendages to be controlled are taken have nonlinear, time-invariant dynamics, with unknown nonlinearities bounded in magnitude from a known, linear, and stable nominal system. We describe the nominal system with an $n$-th order state vector, $\boldsymbol{x}=$ $\left[x_{1} x_{2} \cdots x_{n}\right]^{\mathrm{T}}$, and its dynamics using the state equations

$$
\dot{\boldsymbol{x}}(t)=\boldsymbol{A} \boldsymbol{x}(t)+\boldsymbol{B} u(t)
$$

where $\boldsymbol{A}$ is an $n \times n$ state matrix, $\boldsymbol{B}$ is an $n \times 1$ input matrix, and $u(t)$ is the controlled input to the system.

The real, unknown system is denoted by separate state vector, $\boldsymbol{y}=\left[\begin{array}{llll}y_{1} & y_{2} & \cdots & y_{n}\end{array}\right]^{\mathrm{T}}$, taken to have dynamics of the form

$$
\dot{\boldsymbol{y}}(t)=\left[\boldsymbol{A} \boldsymbol{y}(t)+f_{y}(\boldsymbol{y}(t))\right]+\left[\boldsymbol{B}+f_{u}(\boldsymbol{y}(t))\right] u(t)
$$

where $f_{y}($.$) and f_{u}($.$) are unknown, Lipchitz contin-$ uous, and potentially nonlinear functions of the states. Both $\boldsymbol{x}$ and $\boldsymbol{y}$ are assumed to be asymptotically stable systems.

The system has two possible input values, otherwise described as 'off', $u=0$, and 'on', $u=u_{\max }$, voltages to the actuator, such that

$$
u(t) \in\left\{0, u_{\max }\right\} \forall t
$$

In theory, with sufficient sampling speed on the output displacement, system identification techniques may be used to identify the system model. From there, optimal on-off switching times may be computed to produce desired motions using integer programming or other optimization techniques. However, this optimization is computationally intensive, and the sampling rate needed for effective system identification is high, requiring large sensor power consumption. Thus, a model-free approach to guide motion while using a limited number of sensor measurements is advantageous for micro-robotic servo control. Instead of real-time system identification, the control inputs will be adjusted over multiple iterations of a desired motion.

The first state of the system, $y_{1}$, is measured at a finite number, $Q$, of time points, $t_{1}, t_{2}, \ldots, t_{Q}$, with $t_{Q}$

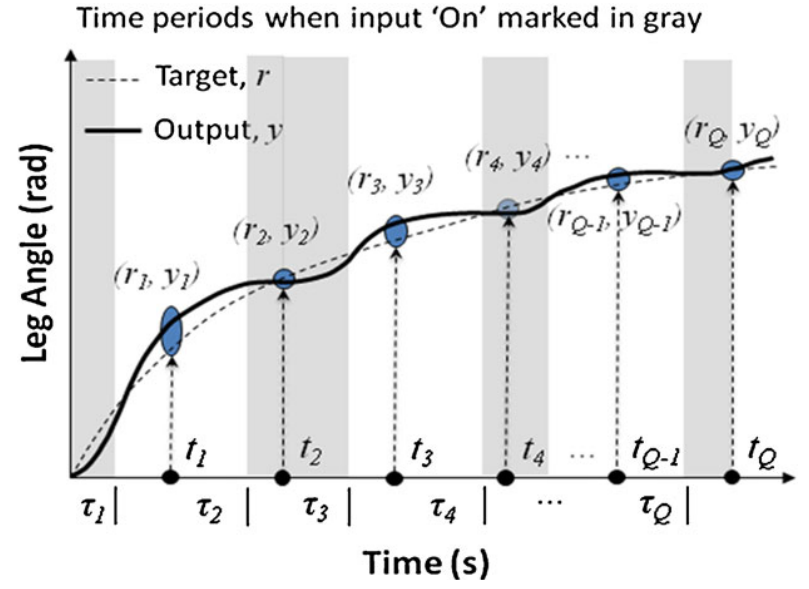

Fig. 2. General definition of switching times and sampling times (in scenario with equal number of switching and sampling instants).

being both the final measurement time and the end of the duration of a single movement by the plant, beginning from initial states $\boldsymbol{y}(0)$. A target output for the system may be prescribed at each measurement time, by references $r_{1}$ to $r_{Q}$, although for the bulk of this paper we will focus on a step-and-hold motion to a single reference level, $r$. The on-off control input, meanwhile, switches at $P$ switching times $\tau_{1}$ to $\tau_{P} \leq t_{Q}$ recorded in vector $\tau$

$$
\begin{aligned}
& \tau=\left[\begin{array}{llll}
\tau_{1} & \tau_{2} & \cdots & \tau_{p}
\end{array}\right] \\
& o \leq \tau_{1} \\
& \tau_{i-1} \leq \tau_{i}, \quad i=2, \ldots, p
\end{aligned}
$$

which, as illustrated in Fig. 2, results in the input to the system being described over time by

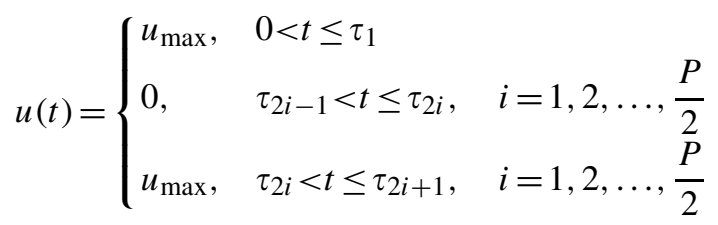

The differential equation (2) is taken to have a real, though unknown, solution, $\boldsymbol{\psi}_{y}$, that may be written as functions of the states at a given starting time, $t_{0}$, the current time, and the input, which may be further simplified to solutions $\psi_{0}$ and $\psi_{1}$ over periods in which the system is subject solely to an 'off' or 'on' input, respectively:

$$
\begin{aligned}
\boldsymbol{y}^{k}(t) & =\boldsymbol{\psi}_{y}\left(t-t_{0}, \boldsymbol{y}^{k}\left(t_{0}\right), u^{k}(t)\right) \\
& = \begin{cases}\psi_{0}\left(t-t_{0}, \boldsymbol{y}^{k}\left(t_{0}\right)\right), & u(t)=0 \forall t>t_{0} \\
\psi_{1}\left(t-t_{1}, \boldsymbol{y}^{k}\left(t_{0}\right)\right), & u(t)=u_{\max } \forall t>t_{0}\end{cases}
\end{aligned}
$$


While in general it is difficult to make statements about the response of an unknown, nonlinear system to a switching input, with certain assumptions about the size of the unknown nonlinearity relative to a nominal system, a number of facts may be concluded about the system response. In contrast, the nominal linear system evolves according to a known state transition matrix, $\Phi$. We combine this with forcing effects to denote the total responses $\Phi_{0}$ and $\Phi_{1}$ as a function of time and initial conditions when the system is purely in an 'off' or 'on' state for a specific period, respectively, as in

$$
\begin{aligned}
& \boldsymbol{x}(t)=\Phi\left(t-t_{0}\right) \boldsymbol{x}\left(t_{0}\right)+\int_{t_{0}}^{t} e^{A(t-s)} \boldsymbol{B} u(t) \mathrm{d} s \\
& = \begin{cases}\Phi\left(t-t_{0}\right) x\left(t_{0}\right), & u=0 \quad \forall t>t_{0} \\
\Phi\left(t-t_{0}\right) x\left(t_{0}\right)+\Omega\left(t-t_{0}\right), & u=u_{\max } \quad \forall t>t_{0}\end{cases}
\end{aligned}
$$

The nonlinear portions of the system are assumed to be bounded as a function of both absolute and relative magnitudes of the states, according to

$$
\begin{aligned}
& \left|\boldsymbol{f}_{y}\left(\boldsymbol{y}^{\prime}(t)\right)-\boldsymbol{f}_{y}(\boldsymbol{y}(t))\right| \leq \boldsymbol{C}_{y}^{\prime}\left|\boldsymbol{y}^{\prime}(t)-\boldsymbol{y}(t)\right| \\
& \left|\boldsymbol{f}_{u}\left(\boldsymbol{y}^{\prime}(t)\right)-\boldsymbol{f}_{u}(\boldsymbol{y}(t))\right| \leq \boldsymbol{C}_{u}^{\prime}\left|\boldsymbol{y}^{\prime}(t)-\boldsymbol{y}(t)\right| \\
& \left|\boldsymbol{f}_{y}(\boldsymbol{y}(t))\right| \leq \boldsymbol{C}_{y}|\boldsymbol{y}(t)| \\
& \left|\boldsymbol{f}_{u}(\boldsymbol{y}(t))\right| \leq \boldsymbol{C}_{u}|\boldsymbol{y}(t)|
\end{aligned}
$$

where $\boldsymbol{C}_{y}, \boldsymbol{C}_{u}, \boldsymbol{C}_{y}^{\prime}$, and $\boldsymbol{C}_{u}^{\prime}$ are matrices with real, non-negative entries, $\boldsymbol{f}_{y}=\left[f_{y 1}, f_{y 2}, \ldots, f_{y n}\right]^{\mathrm{T}}, \boldsymbol{f}_{u}=$ $\left[f_{u 1}, f_{u 2}, \ldots, f_{u n}\right]^{\mathrm{T}}, \quad \boldsymbol{y}=\left[y_{1}, y_{2}, \ldots, y_{n}\right]^{\mathrm{T}}, \quad$ and $\boldsymbol{y}^{\prime}=$ $\left[y_{1}^{\prime}, y_{2}^{\prime}, \ldots, y_{n}^{\prime}\right]^{\mathrm{T}}$.

The nonlinear system, like the nominal linear system, is assumed to be asymptotically stable. When the input is 'off', the equilibrium position is assumed to be $\boldsymbol{y}=0$, while the equilibrium position for an 'on' input is not necessarily known.

Finally, we assume that an upper bound, $\boldsymbol{x}_{\max }$, on the maximum values of the states is known, or

$$
|\boldsymbol{x}|<\boldsymbol{x}_{\max }
$$

This bound does not need to be known very accurately, and is used only to provide a starting point for a numerical integration during convergence analysis.

\section{CONTROLER DESIGN}

Conceptually, the goal of the iterative controller for a micro-robotic leg joint is to drive the joint to a desired output angle or displacement, $r$, with little overshoot or oscillation, despite the availability of only two input levels ('on' and 'off') and relatively sparse position sensor measurements. The controller should use measurements from a previous step or steps to adjust the on-off switching times in the next iteration, and converge over several iterations to a smooth stepping motion. The system is assumed to start each in the equilibrium position, with initial conditions

$$
\boldsymbol{y}^{k}(0)=0, \quad \dot{y}^{k}(0)=0, \ldots,\left.\frac{\mathrm{d}^{n}\left(\boldsymbol{y}^{k}\right)}{\mathrm{d} t^{n}}\right|_{t=0}=0
$$

Each iteration of actuator motion under the iterative adaptive controller has duration $t_{Q}$, with sufficient time before the next iteration to return to the system's equilibrium position in the 'off' state. While the exact model of the system, may be unknown, the known information about the system in (1)-(3) and (7)(9) guides the on-off switching rule formulation for the desired task. During analysis of this adaptation rule, the response of the system in the $K$ th iteration is denoted with a superscript $K$ above the variables of interest, such that the actual response, nominal response, input, and switching time vector, respectively, are denoted by $\boldsymbol{y}^{K}(t), \boldsymbol{x}^{K}(t), u^{K}(t)$, and $\tau^{K}$. Time $t$ is reset to zero at the beginning of each iteration, so all switching times and measurement times are measured relative to the start of the iteration marked.

For specifically controlling a smooth stepping motion of the system, we will adapt only three independent switching times. First, there will be an initial pulse length, $\tau_{p}=\tau_{1}$, that is intended to drive the value of $y$ to a target output value $r$ at the first measurement time $t_{1}$, roughly corresponding to the peak level of the response. The second switching time, $\tau_{s}=\tau_{2}$, regulates the standby time of the first 'off' period, attempting to keep the output at the second measurement equal to that of the first, before entering a periodic on-off switching pattern initiated and regulated by the third independent switching time, $\tau_{d}$. Beginning in this third time period, the input to the system is switched 'on' at a constant frequency and duty cycle determined by $\tau_{d}$, as illustrated in Fig. 3. After a period $t_{Q}$, the input to the system is turned 'off' and the system is then assumed to be allowed sufficient time to return to the equilibrium position with zero ('off') input, and the next iteration begins. This generic approach to on-off control provides a simple method for guiding micro-robotic leg joints to a desired motion, while reducing the number of independent switching times to be adapted to three specific time intervals 


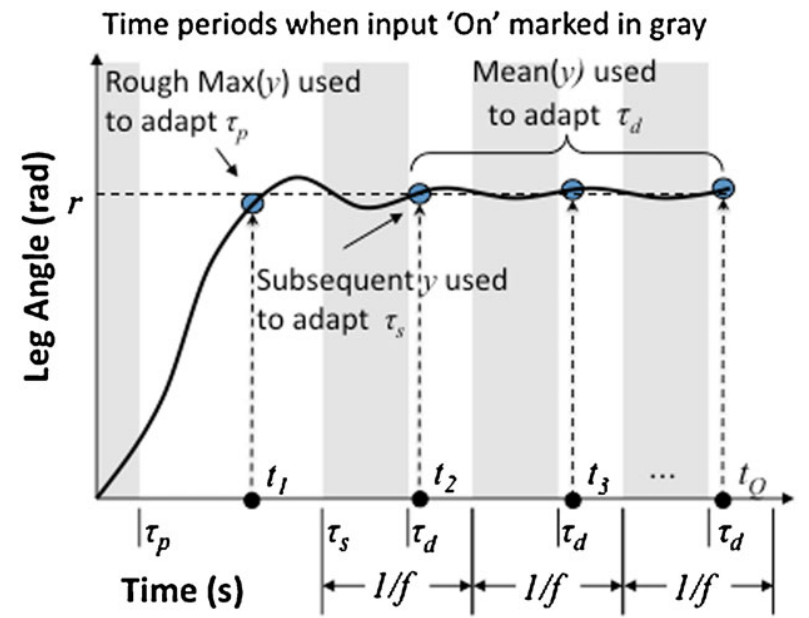

Fig. 3. Definition of switching times and sample times for a specific step controller.

between transitions, each reflecting a phase of step motion.

\subsection{Measurement data}

Since only limited sensor measurements are desired to maintain a relatively slow sampling rate, the target outputs for the resulting motion are limited to certain functions of these few measurements. To perform the stepping motion described above, relevant output measurements are selected to represent common phenomena in dynamic system step responses:

$y_{p}^{K}$ : The first measurement taken, such that $y_{p}^{K}=$ $y_{1}^{K}\left(t_{1}\right)$, corresponding to the approximate peak value of the output in a single iteration of the stepping motion.

$y_{s}^{K}$ : The next sampled value after the apparent peak, $y_{s}^{K}=y_{1}^{K}\left(t_{2}\right)$, when approximately the steadystate 'hold' behavior of the controller will have begun.

$y_{d}^{K}$ : The average value of the remaining measurements before some final end time $t_{Q}$. During this period, an approximately steady state response should occur maintaining the output of the controller near the reference level.

\subsection{Adaptation law}

Given the control parameters to be adjusted, $\tau_{p}, \tau_{s}$, and $\tau_{d}$, and measurements, $y_{p}, y_{s}$, and $y_{d}$, a very simple adaptation law can be implemented to adjust microrobotic leg motion. In this law, the next set of control parameters for each adaptation cycle can be estimated by current control parameters and current performance measurements as follows:

$$
\begin{aligned}
\tau_{p}^{K+1} & =\tau_{p}^{K}+\gamma_{p}\left(r-y_{p}^{K}\right) \\
\tau_{s}^{K+1} & =\tau_{s}^{K}+\gamma_{s}\left(y_{s}^{K}-y_{p}^{K}\right) \\
\tau_{d}^{K+1} & =\tau_{d}^{K}+\gamma_{d}\left(r-y_{d}^{K}\right)
\end{aligned}
$$

where $\gamma_{p}, \gamma_{s}$, and $\gamma_{d}$ are adaptive control constants and obtained by convergence analysis for the proposed controller, which is described in the next section. This adaptation law was selected to imitate proportional control of the measurements of interest, which allows both extremely simple implementation and tractable, though much more complex, convergence analysis. Other adaptation laws could be tested using more complex functions of the measurements, but the adaptation rule in (12) has proven to provide sufficient performance for the target micro-robotic application.

The control law is implemented using the on-off decision rule shown in equation (13).

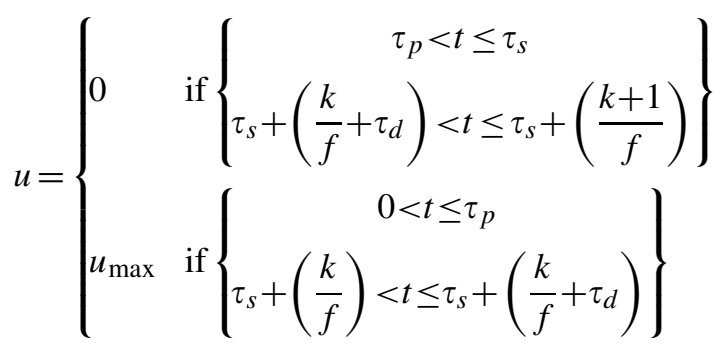

where $f$ is a desired switching frequency in the steadystate phase, selected by the designer, and $k$ is a counting variable for the number of steady-state switches, $k=$ $0,1,2, \ldots, k_{\max }$, occurring before the end time for the iteration, $t_{Q}$.

\section{CONVERGENCE ANALYSIS APPROACH}

\subsection{Requirement for convergence}

The behavior of a system of the type described in (1)-(3) under the adaptation law (12) may be viewed as a discrete time dynamic system, with equilibrium points from (12) of $y_{p}=r, y_{s}=y_{p}$, and $y_{d}=r$. It may be possible for the system to converge to these equilibrium points as $K \rightarrow \infty$ under the given adaptation law if there are some ranges of switching times, $\tau_{p}^{*}, \tau_{s}^{*}$ and $\tau_{d}^{*}$ and maximum allowable changes in switching times, 
$\delta \tau_{p, \max }>0, \delta \tau_{s, \max }>0$, and $\delta \tau_{d, \max }>0$, such that

$$
\begin{aligned}
& \left\{\begin{array} { l } 
{ \tau _ { p } ^ { K } \in \tau _ { p } ^ { * } , 0 < ( \tau _ { p } ^ { K + 1 } - \tau _ { p } ^ { K } = \delta \tau _ { p } ^ { K + 1 } ) < \delta \tau _ { p , \operatorname { m a x } } } \\
{ \tau _ { s } ^ { K } \in \tau _ { s } ^ { * } , 0 < ( \tau _ { s } ^ { K + 1 } - \tau _ { s } ^ { K } = \delta \tau _ { s } ^ { K + 1 } ) < \delta \tau _ { s , \operatorname { m a x } } } \\
{ \tau _ { d } ^ { K } \in \tau _ { d } ^ { * } , 0 < ( \tau _ { d } ^ { K + 1 } - \tau _ { d } ^ { K } = \delta \tau _ { d } ^ { K + 1 } ) < \delta \tau _ { d , \operatorname { m a x } } }
\end{array} \Rightarrow \left\{\begin{array}{l}
y_{p}^{K+1}>y_{p}^{K} \\
y_{s}^{K+1}<y_{s}^{K} \text { or } \\
y_{d}^{K+1}>y_{d}^{K}
\end{array}\right.\right. \\
& \left\{\begin{array} { l } 
{ \tau _ { p } ^ { K } \in \tau _ { p } ^ { * } , - \delta \tau _ { p , \operatorname { m a x } } < ( \tau _ { p } ^ { K + 1 } - \tau _ { p } ^ { K } = \delta \tau _ { p } ^ { K + 1 } ) < 0 } \\
{ \tau _ { s } ^ { K } \in \tau _ { s } ^ { * } , - \delta \tau _ { s , \operatorname { m a x } } < ( \tau _ { s } ^ { K + 1 } - \tau _ { s } ^ { K } = \delta \tau _ { s } ^ { K + 1 } ) < 0 } \\
{ \tau _ { d } ^ { K } \in \tau _ { d } ^ { * } , - \delta \tau _ { d , \operatorname { m a x } } < ( \tau _ { d } ^ { K + 1 } - \tau _ { d } ^ { K } = \delta \tau _ { d } ^ { K + 1 } ) < 0 }
\end{array} \Rightarrow \left\{\begin{array}{l}
y_{p}^{K+1}<y_{p}^{K} \\
y_{s}^{K+1}>y_{s}^{K} \\
y_{d}^{K+1}<y_{d}^{K}
\end{array}\right.\right.
\end{aligned}
$$

If this is the case, then there will also exist some $\gamma_{p}, \gamma_{s}$, and $\gamma_{d}$ small enough to ensure that

$$
\begin{aligned}
& \left|y_{p}^{K+1}-r\right|<\left|y_{p}^{K}-r\right| \\
& \left|y_{s}^{K+1}-y_{p}^{K+1}\right|<\left|y_{s}^{K}-y_{p}^{K}\right| \\
& \left|y_{d}^{K+1}-r\right|<\left|y_{d}^{K}-r\right|
\end{aligned}
$$

for $\tau_{p}^{K} \in \tau_{p}^{*}, \tau_{s}^{K} \in \tau_{s}^{*}$ and $\tau_{d}^{K} \in \tau_{d}^{*}$. Switching times within these ranges are then guaranteed to move the measured outputs closer to their targets in the next time step.

To ensure convergence to the targets themselves, a closed subset of switching time ranges, $\tau_{p}^{* c}, \tau_{s}^{* c}$ and $\tau_{d}^{* c}$, must be identified such that $\tau_{p}^{K+1} \in \tau_{p}^{* c}, \tau_{s}^{K+1} \in \tau_{s}^{* c}$, and $\tau_{d}^{K+1} \in \tau_{d}^{* c}$ if $\tau_{p}^{K} \in \tau_{p}^{* c}, \tau_{s}^{K} \in \tau_{s}^{* c}$, and $\tau_{d}^{K} \in \tau_{d}^{* c}$. In addition, for a given $r, y_{p}=r, y_{s}=y_{p}$, and $y_{d}=r$ must be known to exist for some set of switching times among $\tau_{p}^{* c}, \tau_{s}^{* c}$, and $\tau_{d}^{* c}$. The convergence criteria above are ensuring that a function $J(\boldsymbol{K})$ defined by the measurement errors, $e_{p}, e_{s}$, and $e_{d}$, as

$$
\begin{aligned}
J(e(K)) & =\left|e_{p}(K)\right|+\left|e_{s}(K)\right|+\left|e_{d}(K)\right| \\
& =\left|y_{p}^{K}-r\right|+\left|y_{s}^{K}-y_{p}^{K}\right|+\left|y_{d}^{K}-r\right|
\end{aligned}
$$

is being reduced from step $K$ to $K+1$ due to (15) if $\tau_{p}^{K} \in \tau_{p}^{*}, \tau_{s}^{K} \in \tau_{s}^{*}$ and $\tau_{d}^{K} \in \tau_{d}^{*}$, and that this must further be true in ensuing steps if both the switching time set is closed for a given set of adaptation gains and $r$ may be reached for some set of switching times within the set. In other words, with $\boldsymbol{e}(K)=0$ the equilibrium point for (12), $\boldsymbol{D}$ a closed set of switching times satisfying (14), $\boldsymbol{D}=\left[\begin{array}{lll}\tau_{p}^{* c} & \tau_{s}^{* c} & \tau_{d c}^{* c}\end{array}\right]$, that contains some set of switching times corresponding to $\boldsymbol{e}(K)=0$ in $\boldsymbol{R}^{n}$, and $J: \boldsymbol{D} \rightarrow \boldsymbol{R}$ a continuously differentiable function with respect to $K$, then we can select $\gamma_{p}, \gamma_{s}$, and $\gamma_{d}$ small enough that

$$
\begin{aligned}
& J(0)=0 \text { and } J(e(K))>0 \quad \text { in } \boldsymbol{D}-\{0\} \\
& J(e(K+1))-J(e(K))<0
\end{aligned}
$$

is true throughout $\boldsymbol{D}$, with the second statement in (17) being true due to (15). Then, (16) can be a Lyapunov function and the convergence criteria (14) are ensuring asymptotically stable convergence in a Lyapunov sense within the closed set of switching times.

\subsection{Effects of changes to switching times}

In order to identify regions $\tau_{p}^{* c}, \tau_{s}^{* c}$ and $\tau_{d}^{* c}$ for various potential references, $r$, the known, nominal effects of incremental changes in switching times must be compared to uncertainties in these effects due to the unknown nonlinearities in the system model. To do this, the deviation in possible outputs from the nominal response of the system must first be found. Given the nominal system described by states $\boldsymbol{x}$, and the real, but unknown system described by states $\boldsymbol{y}$, define the error between the systems, $z$, having dynamics

$$
\begin{aligned}
\dot{z}(t)= & \dot{\boldsymbol{y}}(t)-\dot{\boldsymbol{x}}(t) \\
= & \boldsymbol{A y}(t)+f_{y}(\boldsymbol{y}(t))+\boldsymbol{B} u(t)+f_{u}(\boldsymbol{y}(t)) u(t) \\
& -\boldsymbol{A x}(t)-\boldsymbol{B} u(t) \\
= & \boldsymbol{A z}(t)+f_{y}(\boldsymbol{y}(t))+f_{u}(\boldsymbol{y}(t)) u(t)
\end{aligned}
$$

having time response

$$
\begin{aligned}
z(t)= & e^{\boldsymbol{A}\left(t-t_{0}\right)} \boldsymbol{z}\left(t_{0}\right) \\
& +\int_{t_{0}}^{t} e^{\boldsymbol{A}(t-s)}\left[f_{y}(\boldsymbol{y}(s))\right. \\
& \left.+f_{u}(\boldsymbol{y}(s)) u(s)\right] \mathrm{d} s
\end{aligned}
$$

where $t_{0}$ is an arbitrary starting point from which error $z(t)$ is to be calculated. Because the precise error is unknown, the magnitude of error may merely be bounded, with the bound defined as a function of time by $\Delta(t)$,

$$
\begin{aligned}
|z(t)| \leq & \Delta(t)=\left|e^{A\left(t-t_{0}\right)}\right| \Delta\left(t_{0}\right) \\
& +\int_{t_{0}}^{t}\left|e^{A(t-s)}\right|\left[C_{y}(|x(s)|\right. \\
& \left.+\Delta(s))+C_{y}(|x(s)|+\Delta(s)) u(s)\right] \mathrm{d} s
\end{aligned}
$$


Given an upper bound on error at time $t_{0}, \Delta(t)$ may be calculated numerically, as by iterating from a loose initial guess for $|z(t)|$, such as $2 \boldsymbol{x}_{\max }$. This typically results in a bounded $\Delta(t)$ reflecting a growing uncertainty in the true system response compared to the nominal system.

To examine the change in system outputs from one time step to the next, we illustrate our procedure by first considering the change in system response at the 2nd switching time, $\tau_{2}$, which concludes an 'off' period. First, using (6), we may separate the change in response according to

$$
\begin{aligned}
\boldsymbol{y}^{K+1} & \left(\tau_{2}^{K+1}\right)-\boldsymbol{y}^{K}\left(\tau_{2}^{K}\right) \\
= & \psi_{0}\left(\tau_{2}^{K+1}-\tau_{1}^{K+1}, \boldsymbol{y}^{K+1}\left(\tau_{1}^{K+1}\right)\right) \\
& -\psi_{0}\left(\tau_{2}^{K}-\tau_{1}^{K}, \boldsymbol{y}^{K}\left(\tau_{1}^{K}\right)\right) \\
= & \int_{\tau_{2}^{K}-\tau_{1}^{K}}^{\tau_{2}^{K+1}-\tau_{1}^{K+1}} \dot{\psi}_{0}\left(t, \boldsymbol{y}^{K+1}\left(\tau_{1}^{K+1}\right)\right) \mathrm{d} t \\
& +\psi_{0}\left(\tau_{2}^{K}-\tau_{1}^{K}, \boldsymbol{y}^{K+1}\left(\tau_{1}^{K+1}\right)\right) \\
& -\psi_{0}\left(\tau_{2}^{K}-\tau_{1}^{K}, \boldsymbol{y}^{K}\left(\tau_{1}^{K}\right)\right)
\end{aligned}
$$

where the component under the integral reflects the change in the response due to the change in the duration of the 'off' period, and the remaining terms reflect the influence of the change in initial conditions for the 'off' period due to changes in previous time steps (in this case, $\tau_{1}$ ). At times, it is more convenient to express this shift using the general solution to dynamics from (6) as

$$
\begin{aligned}
\boldsymbol{y}^{K+1} & \left(\tau_{2}^{K+1}\right)-\boldsymbol{y}^{K}\left(\tau_{2}^{K}\right) \\
= & \int_{\tau_{2}^{K}+\tau_{1}^{K+1}-\tau_{1}^{K}}^{\tau_{2}^{K+1}} \dot{\psi}_{0}\left(t-\tau_{1}^{K+1}, \boldsymbol{y}^{K+1}\left(\tau_{1}^{K+1}\right)\right) \mathrm{d} t \\
& +\psi_{0}\left(\tau_{2}^{K}-\tau_{1}^{K}, \boldsymbol{y}^{K+1}\left(\tau_{1}^{K+1}\right)\right) \\
& -\psi_{0}\left(\tau_{2}^{K}-\tau_{1}^{K}, \boldsymbol{y}^{K}\left(\tau_{1}^{K}\right)\right) \\
= & \int_{\tau_{2}^{K}+\delta \tau_{1}^{K+1}}^{\tau_{2}^{K+1}} \dot{\psi}_{y}\left(t-\tau_{1}^{K+1}, \boldsymbol{y}^{K+1}\left(\tau_{1}^{K+1}\right), u^{k+1}(t)\right) \mathrm{d} t \\
& +\psi_{0}\left(\tau_{2}^{K}-\tau_{1}^{K}, \boldsymbol{y}^{K+1}\left(\tau_{1}^{K+1}\right)\right) \\
& -\psi_{0}\left(\tau_{2}^{K}-\tau_{1}^{K}, \boldsymbol{y}^{K}\left(\tau_{1}^{K}\right)\right)
\end{aligned}
$$

Inserting dynamics from (2) into the integral portion of (22) and separating it) into nominal and error compo- nents and using (6) and (7) gives

$$
\begin{aligned}
& \boldsymbol{y}^{K+1}\left(\tau_{2}^{K+1}\right)-\boldsymbol{y}^{K}\left(\tau_{2}^{K}\right) \\
& =\left.\int_{\tau_{2}^{K}+\delta \tau_{1}^{K+1}}^{\tau_{2}^{K+1}} \dot{y}^{K+1}(t)\right|_{t_{0}=\tau_{1}^{K+1}} \mathrm{~d} t \\
& +\psi_{0}\left(\tau_{2}^{K}-\tau_{1}^{K}, \boldsymbol{y}^{K+1}\left(\tau_{1}^{K+1}\right)\right) \\
& -\psi_{0}\left(\tau_{2}^{K}-\tau_{1}^{K}, \boldsymbol{y}^{K}\left(\tau_{1}^{K}\right)\right) \\
& =\left.\int_{\tau_{2}^{K}+\delta \tau_{1}^{K+1}}^{\tau_{2}^{K+1}} \dot{\boldsymbol{x}}^{K+1}(t)\right|_{t_{0}=\tau_{1}^{K+1}} \mathrm{~d} t \\
& +\int_{\tau_{2}^{K}+\delta \tau_{1}^{K+1}}^{\tau_{2}^{K+1}}\left[\boldsymbol{A}\left(\boldsymbol{y}^{K+1}(t)-\boldsymbol{x}^{K+1}(t)\right)\right. \\
& +\boldsymbol{f}_{y}\left(\boldsymbol{y}^{K+1}(t)\right) \\
& \left.+\boldsymbol{f}_{u}\left(\boldsymbol{y}^{K+1}(t)\right) u^{K+1}(t)\right]\left.\right|_{t_{0}=\tau_{1}^{K+1}} \mathrm{~d} t \\
& +\psi_{0}\left(\tau_{2}^{K}-\tau_{1}^{K}, \boldsymbol{y}^{K+1}\left(\tau_{1}^{K+1}\right)\right) \\
& -\psi_{0}\left(\tau_{2}^{K}-\tau_{1}^{K}, \boldsymbol{y}^{K}\left(\tau_{1}^{K}\right)\right)
\end{aligned}
$$

from which nominal dynamics under the integral may be used to create nominal and error components for the remainder of the expression,

$$
\begin{aligned}
& \boldsymbol{y}^{K+1}\left(\tau_{2}^{K+1}\right)-\boldsymbol{y}^{K}\left(\tau_{2}^{K}\right) \\
& =\left.\boldsymbol{x}^{K+1}\left(\tau_{2}^{K+1}\right)\right|_{t_{0}=\tau_{1}^{K+1}} \\
& -\left.\boldsymbol{x}^{K+1}\left(\tau_{2}^{K+1}+\delta \tau_{1}^{K+1}\right)\right|_{t_{0}=\tau_{1}^{K+1}} \\
& +\int_{\tau_{2}^{K}+\delta \tau_{1}^{K+1}}^{\tau_{2}^{K+1}}\left[\boldsymbol{A}\left(\boldsymbol{y}^{K+1}(t)-\boldsymbol{x}^{K+1}(t)\right)\right. \\
& +\boldsymbol{f}_{y}\left(\boldsymbol{y}^{K+1}(t)\right) \\
& \left.+\boldsymbol{f}_{u}\left(\boldsymbol{y}^{K+1}(t)\right) u^{K+1}(t)\right]\left.\right|_{t_{0}=\tau_{1}^{K+1}} \mathrm{~d} t \\
& +\psi_{0}\left(\tau_{2}^{K}-\tau_{1}^{K}, \boldsymbol{y}^{K+1}\left(\tau_{1}^{K+1}\right)\right) \\
& -\psi_{0}\left(\tau_{2}^{K}-\tau_{1}^{K}, \boldsymbol{y}^{K}\left(\tau_{1}^{K}\right)\right) \\
& =\Phi\left(\tau_{2}^{K+1}-\tau_{1}^{K+1}\right) x^{K+1}\left(\tau_{1}^{K+1}\right) \\
& -\Phi\left(\tau_{2}^{K}-\tau_{1}^{K}\right) \boldsymbol{x}^{K}\left(\tau_{1}^{K}\right) \\
& +\int_{\tau_{2}^{K}+\delta \tau_{1}^{K+1}}^{\tau_{2}^{K+1}}\left[\boldsymbol{A}\left(\boldsymbol{y}^{K+1}(t)-\boldsymbol{x}^{K+1}(t)\right)\right. \\
& +\boldsymbol{f}_{y}\left(\boldsymbol{y}^{K+1}(t)\right)
\end{aligned}
$$




$$
\begin{aligned}
& \left.+\boldsymbol{f}_{u}\left(\boldsymbol{y}^{K+1}(t)\right) u^{K+1}(t)\right]\left.\right|_{t_{0}=\tau_{1}^{K+1}} \mathrm{~d} t \\
& +\psi_{0}\left(\tau_{2}^{K}-\tau_{1}^{K}, \boldsymbol{y}^{K+1}\left(\tau_{1}^{K+1}\right)\right) \\
& -\Phi\left(\tau_{2}^{K}-\tau_{1}^{K}\right) \boldsymbol{x}^{K+1}\left(\tau_{1}^{K+1}\right) \\
& -\psi_{0}\left(\tau_{2}^{K}-\tau_{1}^{K}, \boldsymbol{y}^{K}\left(\tau_{1}^{K}\right)\right) \\
& +\Phi\left(\tau_{2}^{K}-\tau_{1}^{K}\right) \boldsymbol{x}^{K}\left(\tau_{1}^{K}\right) \\
= & \boldsymbol{x}^{K+1}\left(\tau_{2}^{K+1}\right)-\boldsymbol{x}^{K}\left(\tau_{2}^{K}\right) \\
& +\int_{\tau_{2}^{K}+\delta \tau_{1}^{K+1}}^{\tau_{2}^{K+1}}\left[\boldsymbol{A}\left(\boldsymbol{y}^{K+1}(t)-\boldsymbol{x}^{K+1}(t)\right)\right. \\
& +\boldsymbol{f}_{\boldsymbol{y}}\left(\boldsymbol{y}^{K+1}(t)\right) \\
& \left.+\boldsymbol{f}_{u}\left(\boldsymbol{y}^{K+1}(t)\right) u^{K+1}(t)\right]\left.\right|_{t_{0}=\tau_{1}^{K+1}} \mathrm{~d} t \\
& +\left[\psi_{0}\left(\tau_{2}^{K}-\tau_{1}^{K}, \boldsymbol{y}^{K+1}\left(\tau_{1}^{K+1}\right)\right)\right. \\
& -\Phi\left(\tau_{2}^{K}-\tau_{1}^{K}\right) \boldsymbol{x}^{K+1}\left(\tau_{1}^{K+1}\right) \\
& -\psi_{0}\left(\tau_{2}^{K}-\tau_{1}^{K}, \boldsymbol{y}^{K}\left(\tau_{1}^{K}\right)\right) \\
& \left.+\Phi\left(\tau_{2}^{K}-\tau_{1}^{K}\right) \boldsymbol{x}^{K}\left(\tau_{1}^{K}\right)\right]
\end{aligned}
$$

In the above equation, the change in nominal response, $\boldsymbol{x}^{K+1}\left(\tau_{2}^{K+1}\right)-\boldsymbol{x}^{K}\left(\tau_{2}^{K}\right)$ is easily calculated, while the remaining terms may be bounded. First,

$$
\begin{aligned}
& \int_{\tau_{2}^{K}+\delta \tau_{1}^{K+1}}^{\tau_{2}^{K+1}}\left[\boldsymbol{A}\left(\boldsymbol{y}^{K+1}(t)-\boldsymbol{x}^{K+1}(t)\right)+\boldsymbol{f}_{y}\left(\boldsymbol{y}^{K+1}(t)\right)\right. \\
& \left.+\boldsymbol{f}_{u}\left(\boldsymbol{y}^{K+1}(t)\right) u^{K+1}(t)\right]\left.\right|_{t_{0}=\tau_{1}^{K+1} \mathrm{~d} t} \\
& \quad \leq \max _{\left(\tau_{2}^{K}+\delta \tau_{1}^{K+1}, \tau_{2}^{K+1}\right)}\left[|\boldsymbol{A}| \Delta^{K+1}(t)\right. \\
& +\boldsymbol{C}_{y}\left(\left|\boldsymbol{x}^{K+1}(t)\right|+\Delta^{K+1}(t)\right) \\
& \left.+\boldsymbol{C}_{u}\left(\left|\boldsymbol{x}^{K+1}(t)\right|+\Delta^{K+1}(t)\right) u^{K+1}(t)\right] \\
& \cdot\left|\delta \tau_{2}^{K+1}-\delta \tau_{1}^{K+1}\right| \equiv \Delta_{D T}^{K+1}\left(\tau_{2}^{K+1}\right)
\end{aligned}
$$

with the right-side portion of (25) in brackets acting as an upper bound on the rate of change of error between the nominal and true response over the change in 'off' duration, and the quantity $\Delta_{D T}(t)$ denoting an upper bound on error in the estimated change in response due to change in duration of the current 'on' or 'off' period at time $t$.

The influence of changing initial conditions for the 'off' period is more complicated to bound, following a procedure similar to that for bounding $z(t)$. A second set of error states, $z_{I C}$, is defined as

$$
\begin{aligned}
z_{I C}^{K+1}= & \psi_{j}\left(t-t_{0}, \boldsymbol{y}^{K+1}\left(t_{0}\right)\right)-\Phi\left(t-t_{0}\right) \boldsymbol{x}^{K+1}\left(t_{0}\right) \\
& -\psi_{j}\left(t-t_{0}, \boldsymbol{y}^{K}\left(t_{0}\right)\right)+\Phi\left(t-t_{0}\right) \boldsymbol{x}^{K}\left(t_{0}\right)
\end{aligned}
$$

to evaluate the difference in two responses beginning from different initial conditions, with $j=0$ or 1 depending on whether the system is in the 'on' or 'off' case over the current time period. This error evolves according to dynamics (18)

$$
\begin{aligned}
\dot{z}_{I C}^{K+1}(t)= & \boldsymbol{A} \psi_{j}\left(t-t_{0}, \boldsymbol{y}^{K+1}\left(t_{0}\right)\right) \\
& +f_{y}\left(\psi_{j}\left(t-t_{0}, \boldsymbol{y}^{K+1}\left(t_{0}\right)\right)\right)+\boldsymbol{B} u^{K+1}(t) \\
& +f_{u}\left(\psi_{j}\left(t-t_{0}, \boldsymbol{y}^{K+1}\left(t_{0}\right)\right)\right) u^{K+1}(t) \\
& -\boldsymbol{A} \Phi\left(t-t_{0}\right) \boldsymbol{x}^{K+1}\left(t_{0}\right)-\boldsymbol{B} u^{K+1}(t) \\
& -\boldsymbol{A} \psi_{j}\left(t-t_{0}, \boldsymbol{y}^{K}\left(t_{0}\right)\right) \\
& +f_{y}\left(\psi_{j}\left(t-t_{0}, \boldsymbol{y}^{K}\left(t_{0}\right)\right)\right)+\boldsymbol{B} u^{K}(t) \\
& +f_{u}\left(\psi_{j}\left(t-t_{0}, \boldsymbol{y}^{K}\left(t_{0}\right)\right)\right) u^{K}(t) \\
& -\boldsymbol{A} \Phi\left(t-t_{0}\right) \boldsymbol{x}^{K}\left(t_{0}\right)-\boldsymbol{B} u^{K}(t) \\
= & \boldsymbol{A} z_{I C}^{K+1}(t)+f_{y}\left(\psi_{j}\left(t-t_{0}, \boldsymbol{y}^{K+1}\left(t_{0}\right)\right)\right) \\
& -f_{y}\left(\psi_{j}\left(t-t_{0}, \boldsymbol{y}^{K}\left(t_{0}\right)\right)\right) \\
& +f_{u}\left(\psi_{j}\left(t-t_{0}, \boldsymbol{y}^{K+1}\left(t_{0}\right)\right)\right) u^{K+1}(t) \\
& -f_{u}\left(\psi_{j}\left(t-t_{0}, \boldsymbol{y}^{K}\left(t_{0}\right)\right)\right) u^{K}(t)
\end{aligned}
$$

and it may be noted that within a given 'on' or 'off' period in the sequence of inputs, $u^{K}(t)$ and $u^{K+1}(t)$ will have the same value. An upper bound on error due to a change in initial conditions for the current 'on' or 'off' period, $\Delta_{I C}(\mathrm{t})$, may be applied that is obtained numerically from

$$
\begin{aligned}
\left|z_{I C}^{K+1}(t)\right| \leq & \Delta_{I C}^{K+1}(t)=\left|e^{\boldsymbol{A}\left(t-t_{0}\right)}\right| \Delta_{I C}^{K+1}\left(t_{0}\right) \\
& +\int_{t_{0}}^{t}\left|e^{\boldsymbol{A}(t-s)}\right|\left[\boldsymbol { C } _ { y } \left(\left|\boldsymbol{x}^{K+1}(s)-\boldsymbol{x}^{K}(s)\right|\right.\right. \\
& \left.+\Delta_{I C}^{K+1}(s)\right)+\boldsymbol{C}_{u}\left(\left|\boldsymbol{x}^{K+1}(s)-\boldsymbol{x}^{K}(s)\right|\right. \\
& \left.\left.+\Delta_{I C}^{K+1}(s)\right) u(s)\right] \mathrm{d} s .
\end{aligned}
$$

Applying (26)-(28) to remaining term of (24),

$$
\begin{aligned}
\mid \psi_{0}\left(\tau_{2}^{K}-\tau_{1}^{K}, \boldsymbol{y}^{K+1}\left(\tau_{1}^{K+1}\right)\right)-\Phi\left(\tau_{2}^{K}-\tau_{1}^{K}\right) \boldsymbol{x}^{K+1}\left(\tau_{1}^{K+1}\right) \\
\quad-\psi_{0}\left(\tau_{2}^{K}-\tau_{1}^{K}, \boldsymbol{y}^{K}\left(\tau_{1}^{K}\right)\right)-\Phi\left(\tau_{2}^{K}-\tau_{1}^{K}\right) \boldsymbol{x}^{K}\left(\tau_{1}^{K}\right) \mid \\
\leq\left|e^{\boldsymbol{A}\left(\tau_{2}^{K}-\tau_{1}^{K}\right)}\right| \Delta_{I C}^{K+1}\left(\tau_{1}^{K+1}\right)
\end{aligned}
$$




$$
\begin{aligned}
& +\int_{\tau_{1}^{K}}^{\tau_{2}^{K}}\left(\begin{array}{c}
\left|e^{\boldsymbol{A}(t-s)}\right|\left[\boldsymbol { C } _ { y } \left(\left|\boldsymbol{x}^{K+1}(s)-\boldsymbol{x}^{K}(s)\right|\right.\right. \\
\left.+\Delta_{I C}^{K+1}(s)\right)+\boldsymbol{C}_{u}\left(\mid \boldsymbol{x}^{K+1}(s)\right. \\
\left.\left.-\boldsymbol{x}^{K}(s) \mid+\Delta_{I C}^{K+1}(s)\right) u(s)\right]
\end{array}\right) \mathrm{d} s \\
& =\Delta_{I C}^{K+1}\left(\tau_{2}^{K}\right)
\end{aligned}
$$

where the initial error for this period, $\Delta_{I C}^{K+1}\left(\tau_{1}^{K+1}\right)$, is any error due to change in the previous switching times, which in this case is only the effect of the change in 'on' time, $\tau_{1}^{K+1}$ versus $\tau_{1}^{K}$. The bound here, $\Delta_{I C}^{K+1}\left(\tau_{2}^{K}\right)$ is the maximum effect of changes in initial conditions for the current 'on' or 'off' phase of motion, assuming the previous switching time were used during this phase (while effects of the change in duration of this 'on' or 'off' phase on overall behavior is captured in (25)). Therefore, using same procedure of (23)-(25) and assumption (11), we can obtain a relation,

$$
\begin{aligned}
& \Delta_{I C}^{K+1}\left(\tau_{1}^{K+1}\right) \\
& =\left|\boldsymbol{y}^{K+1}\left(\tau_{1}^{K+1}\right)-\boldsymbol{y}^{K}\left(\tau_{1}^{K}\right)\right|_{\max } \\
& =\left|\begin{array}{l}
\boldsymbol{x}^{K+1}\left(\tau_{1}^{K+1}\right)-\boldsymbol{x}^{K}\left(\tau_{1}^{K}\right)+\int_{\tau_{1}^{K}+\delta \tau_{0}^{K+1}}^{\tau_{1}^{K+1}}\left[\boldsymbol { A } \left(\boldsymbol{y}^{K+1}(t)\right.\right. \\
\left.\quad-\boldsymbol{x}^{K+1}(t)\right)+\boldsymbol{f}_{y}\left(\boldsymbol{y}^{K+1}(t)\right) \\
\left.\quad+\boldsymbol{f}_{u}\left(\boldsymbol{y}^{K+1}(t)\right) u^{K+1}(t)\right]\left.\right|_{t_{0}=0} \mathrm{~d} t \\
\quad+\left[\psi_{1}\left(\tau_{1}^{K}-0, \boldsymbol{y}^{K+1}\left(\tau_{0}^{K+1}\right)\right)\right. \\
\quad-\Phi\left(\tau_{1}^{K}-0\right) \boldsymbol{x}^{K+1}\left(\tau_{0}^{K+1}\right) \\
\left.-\psi_{1}\left(\tau_{1}^{K}-0, \boldsymbol{y}^{K}\left(\tau_{0}^{K}\right)\right)+\Phi\left(\tau_{1}^{K}-0\right) \boldsymbol{x}^{K}\left(\tau_{0}^{K}\right)\right]
\end{array}\right|_{\max } \\
& \mid \boldsymbol{x}^{K+1}\left(\tau_{2}^{K+1}\right)-\boldsymbol{x}^{K}\left(\tau_{2}^{K}\right)+\int_{\tau_{1}^{K}}^{\tau_{1}^{K+1}}\left[\boldsymbol { A } \left(\boldsymbol{y}^{K+1}(t) \mid\right.\right. \\
& \left.-x^{K+1}(t)\right)+f_{y}\left(y^{K+1}(t)\right) \\
& \left.=\quad+\boldsymbol{f}_{u}\left(\boldsymbol{y}^{K+1}(t)\right) u^{K+1}(t)\right]\left.\right|_{t_{0}=0} \mathrm{~d} t \\
& +\left[\psi_{1}\left(\tau_{1}^{K}, \boldsymbol{y}^{K+1}(0)\right)\right. \\
& -\Phi\left(\tau_{1}^{K}\right) \boldsymbol{x}^{K+1}(0)-\psi_{1}\left(\tau_{1}^{K}, \boldsymbol{y}^{K}(0)\right) \\
& \left.+\Phi\left(\tau_{1}^{K}\right) \boldsymbol{x}^{K}(0)\right] \\
& =\left|\begin{array}{l}
\boldsymbol{x}^{K+1}\left(\tau_{2}^{K+1}\right)-\boldsymbol{x}^{K}\left(\tau_{2}^{K}\right) \\
+\int_{\tau_{1}^{K}}^{\tau_{1}^{K+1}}\left[\boldsymbol{A}\left(\boldsymbol{y}^{K+1}(t)-\boldsymbol{x}^{K+1}(t)\right)\right. \\
+\boldsymbol{f}_{y}\left(\boldsymbol{y}^{K+1}(t)\right) \\
\left.+\boldsymbol{f}_{u}\left(\boldsymbol{y}^{K+1}(t)\right) u^{K+1}(t)\right]\left.\right|_{t_{0}=0} \mathrm{~d} t
\end{array}\right|_{\max }
\end{aligned}
$$

$$
\begin{aligned}
= & \max _{\left(\tau_{1}^{K}, \tau_{1}^{K+1}\right)}\left[|\boldsymbol{A}| \Delta^{K+1}(t)+\boldsymbol{C}_{y}\left(\left|\boldsymbol{x}^{K+1}(t)\right|\right.\right. \\
& \left.+\Delta^{K+1}(t)\right)+\boldsymbol{C}_{u}\left(\left|\boldsymbol{x}^{K+1}(t)\right|\right. \\
& \left.\left.+\Delta^{K+1}(t)\right) u^{K+1}(t)\right] \cdot\left|\delta \tau_{1}^{K+1}\right| \\
\equiv & \Delta_{D T}^{K+1}\left(\tau_{1}^{K+1}\right)
\end{aligned}
$$

that reflects the fact that the initical conditions for the first 'on' step are constant, but in later time steps becomes the cumulative effect of switching time changes at previous transitions, or in other words, for the $Q$-th switching time,

$$
\begin{aligned}
& \Delta_{D T}^{K+1}\left(\tau_{Q}^{K+1}\right) \\
& \leq \max _{\left(\tau_{Q}^{K}+\delta \tau_{Q-1}^{K+1}, \tau_{Q}^{K}\right)}\left[\begin{array}{c}
|\boldsymbol{A}| \Delta^{K+1}(t)+\boldsymbol{C}_{y}\left(\left|\boldsymbol{x}^{K+1}(t)\right|\right. \\
\left.+\Delta^{K+1}(t)\right)+\boldsymbol{C}_{u}\left(\left|\boldsymbol{x}^{K+1}(t)\right|\right. \\
\left.+\Delta^{K+1}(t)\right) u(t)
\end{array}\right] \\
& \times\left|\delta \tau_{Q}^{K+1}-\delta \tau_{Q-1}^{K+1}\right| \\
& \Delta_{I C}^{K+1}\left(\tau_{Q}^{K}\right) \\
& \leq\left[\sum_{q=1}^{Q-1}\left|e^{A\left(\tau_{Q}^{K}-\tau_{q}^{K}\right)}\right|\left(\Delta_{D T}^{K+1}\left(\tau_{q}^{K+1}\right)+\Delta_{I C}^{K+1}\left(\tau_{q}^{K}\right)\right)\right] \\
& +\int_{\tau_{Q-1}^{K}}^{\tau_{Q}^{K}}\left|e^{A(t-s)}\right| \\
& \times\left[\begin{array}{c}
\boldsymbol{C}_{y}\left(\left|\boldsymbol{x}^{K+1}(s)-\boldsymbol{x}^{k}(s)\right|\right. \\
\left.+\Delta_{I C}^{K+1}(s)\right)+\boldsymbol{C}_{u}\left(\mid \boldsymbol{x}^{K+1}(s)\right. \\
\left.-\boldsymbol{x}^{k}(s) \mid+\Delta_{I C}^{K+1}(s)\right) u(s)
\end{array}\right] \mathrm{d} s
\end{aligned}
$$

\subsection{Application to convergence analysis}

At a measurement point, which occurs at the same time in each iteration, the most recent switching time must be identified, denoted by index $Q_{p}$, in order to find uncertainty in the effects of the changes in switching times from iteration $K$ to iteration $K+1$, becoming

$$
\begin{aligned}
& Q_{p}=\max \left(q \mid \tau_{q}<t_{p}\right) \\
& \Delta_{D T}^{K+1}\left(t_{p}\right) \leq \max _{\left(t_{p}+\delta \tau_{Q_{p}}^{K+1}, t_{p}\right)}
\end{aligned}
$$




$$
\begin{aligned}
& {\left[\begin{array}{c}
|\boldsymbol{A}| \Delta^{K+1}(t) \\
+\boldsymbol{C}_{y}\left(\left|\boldsymbol{x}^{K+1}(t)\right|\right. \\
\left.+\Delta^{K+1}(t)\right) \\
+\boldsymbol{C}_{u}\left(\left|\boldsymbol{x}^{K+1}(t)\right|\right. \\
\left.+\Delta^{K+1}(t)\right) u(t)
\end{array}\right]\left|\delta \tau_{Q_{p}}^{K+1}\right|} \\
& \Delta_{I C}^{K+1}\left(t_{p}\right) \leq\left[\sum _ { q = 1 } ^ { Q _ { p } } | e ^ { A ( t _ { p } - \tau _ { q } ^ { K } ) } | \left(\Delta_{D T}^{K+1}\left(\tau_{q}^{K+1}\right)\right.\right. \\
& \left.\left.+\Delta_{I C}^{K+1}\left(\tau_{q}^{K}\right)\right)\right] \\
& +\int_{\tau_{Q_{p}}^{K}}^{t_{p}}\left|e^{A(t-s)}\right| \\
& \times\left[\begin{array}{c}
\boldsymbol{C}_{y}\left(\left|\boldsymbol{x}^{K+1}(s)-\boldsymbol{x}^{k}(s)\right|\right. \\
\left.+\Delta_{I C}^{K+1}(s)\right) \\
+\boldsymbol{C}_{u}\left(\left|\boldsymbol{x}^{K+1}(s)-\boldsymbol{x}^{k}(s)\right|\right. \\
\left.+\Delta_{I C}^{K+1}(s)\right) u(s)
\end{array}\right] \mathrm{d} s
\end{aligned}
$$

Thus, (32) gives the uncertainty in the change in output at the measurement points from one iteration to the next, which may be compared with the nominal change in output to determine whether $y_{p}, y_{s}$, and $y_{d}$ are certain to move in the desired directions. In other words, combinations of $\tau_{p}, \tau_{s}, \tau_{d}$, are evaluated as to whether

$$
\left\{\begin{aligned}
x_{p}^{K+1}> & x_{p}^{K} \&\left|x_{p}^{K+1}-x_{p}^{K}\right|>\Delta_{D T}^{K+1}\left(t_{1}\right) \\
x_{s}^{K+1}< & x_{s}^{K} \&\left|x_{s}^{K+1}-x_{s}^{K}\right|>\Delta_{D T}^{K+1}\left(t_{2}\right) \\
& +\Delta_{I C}^{K+1}\left(t_{2}\right) \\
x_{d}^{K+1}> & x_{d}^{K} \&\left|x_{d}^{K+1}-x_{d}^{K}\right|>\frac{1}{Q+1} \\
& \times \sum_{q=2}^{Q}\left(\Delta_{D T}^{K+1}\left(t_{q}\right)+\Delta_{I C}^{K+1}\left(t_{q}\right)\right)
\end{aligned}\right.
$$

for some $\delta \tau_{p}>0, \delta \tau_{s}>0, \delta \tau_{d}>0$, where $x_{p}, x_{s}$, and $x_{d}$ are defined in the same manner as $y_{p}, y_{s}$, and $y_{d}$, but for the nominal states, $\boldsymbol{x}$. If (33) is satisfied for some candidate switching times, then the behavior of the true response is will satisfy (14) for at least these switching times and shifts in switching times.

To create a closed set of $\tau_{p}, \tau_{s}$ and $\tau_{d}$ in which the measured outputs to move towards their targets, a second criterion must be applied, based on a specific reference $r$ and set of adaptation gains, that requires

$$
\begin{aligned}
& \tau_{p}^{K}+\gamma_{p}\left(r-x_{p}^{K}+\Delta^{K}\left(t_{1}\right)\right) \in \tau_{p}^{* c} \\
& \& \tau_{p}^{K}+\gamma_{p}\left(r-x_{p}^{K}-\Delta^{K}\left(t_{1}\right)\right) \in \tau_{p}^{* c} \\
& \tau_{s}^{K}+\gamma_{s}\left(x_{s}^{K}-x_{p}^{K}+\Delta^{K}\left(t_{2}\right)+\Delta^{K}\left(t_{1}\right)\right) \in \tau_{s}^{* c} \\
& \& \tau_{s}^{K}+\gamma_{s}\left(x_{s}^{K}-x_{p}^{K}-\Delta^{K}\left(t_{2}\right)-\Delta^{K}\left(t_{1}\right)\right) \in \tau_{s}^{* c} \\
& \tau_{d}^{K}+\gamma_{d}\left(r-x_{d}^{K}+\frac{1}{Q-1} \sum_{q=1}^{Q} \Delta^{K}\left(t_{q}\right)\right) \in \tau_{d}^{* c} \\
& \& \tau_{d}^{K}+\gamma_{d}\left(r-x_{d}^{K}-\frac{1}{Q-1} \sum_{q=1}^{Q} \Delta^{K}\left(t_{q}\right)\right) \in \tau_{d}^{* c}
\end{aligned}
$$

Ranges of switching times $\tau_{p}^{* c}, \tau_{s}^{* c}$ and $\tau_{d}^{*}$ whose content satisfy both (33) and (34) for a given reference, $r$, define a region in which the measured outputs will converge to their target values. One necessary condition (though not sufficient, alone) for a region satisfying (34) to exist is that within a continuous subset of $\tau_{p}^{*}, \tau_{s}^{*}$ and $\tau_{d}^{*}$ there exists a $\tau_{p}$, $\tau_{s}$ and $\tau_{d}$ resulting in $x_{p}+\Delta\left(t_{1}\right)<r, x_{s}+\Delta\left(t_{2}\right)<r$, and $x_{d}+\frac{1}{Q-1} \sum_{q=1}^{Q} \Delta\left(t_{q}\right)<r$, and some other $\tau_{p}, \tau_{s}$ and $\tau_{d}$ resulting in $x_{p}-\Delta\left(t_{1}\right)>r, x_{s}-\Delta\left(t_{2}\right)>r$, and $x_{d}-\frac{1}{Q-1} \sum_{q=1}^{Q} \Delta\left(t_{q}\right)>r$. In other words, it is not possible to guarantee that switching times will remain within a closed region if it is not guaranteed to contain switching time selections resulting in outputs below and above the final reference value. While this fact alone may not guarantee convergence, it can be useful for initial evaluation of reference values that may work, by choosing

$$
\begin{aligned}
& r<\tau_{\tau_{p}, \tau_{s}, \tau_{d} \in \tau_{p}^{*}, \tau_{s}^{*}, \tau_{d}^{*}}\left(\operatorname { m i n } \left(x_{p}-\Delta\left(t_{1}\right), x_{s}-\Delta\left(t_{2}\right),\right.\right. \\
& \left.\left.x_{d}-\frac{1}{Q-1} \sum_{q=1}^{Q} \Delta\left(t_{q}\right)\right)\right) \\
& r>\min _{\tau_{p}, \tau_{s}, \tau_{d} \in \tau_{p}^{*}, \tau_{s}^{*}, \tau_{d}^{*}}\left(\operatorname { m a x } \left(x_{p}+\Delta\left(t_{1}\right), x_{s}+\Delta\left(t_{2}\right),\right.\right. \\
& \left.\left.x_{d}+\frac{1}{Q-1} \sum_{q=1}^{Q} \Delta\left(t_{q}\right)\right)\right)
\end{aligned}
$$




\section{CONVERGENCE ANALYSIS FOR A SECOND-ORDER MICROACTUTOR}

To illustrate the use of the convergence analysis in Section IV, and to prove convergence to certain values of $r$ for the experimental system used in system validation, convergence analysis is performed for a 2 ndorder piezoelectric microactuator. The nominal actuator model is

$$
\begin{aligned}
{\left[\begin{array}{l}
\dot{x}_{1}(t) \\
\dot{x}_{2}(t)
\end{array}\right]=} & {\left[\begin{array}{cc}
0 & 1 \\
-38590 & -12.1
\end{array}\right]\left[\begin{array}{l}
x_{1}(t) \\
x_{2}(t)
\end{array}\right] } \\
& +\left[\begin{array}{c}
0 \\
32300
\end{array}\right] u(t)
\end{aligned}
$$

and the nonlinear model has the form

$$
\begin{aligned}
{\left[\begin{array}{l}
\dot{y}_{1}(t) \\
\dot{y}_{2}(t)
\end{array}\right]=} & {\left[\begin{array}{cc}
0 & 1 \\
-38590 & -12.1
\end{array}\right]\left[\begin{array}{l}
y_{1}(t) \\
y_{2}(t)
\end{array}\right] } \\
& +\left[\begin{array}{cc}
0 & 0 \\
f_{y 1}\left(y_{1}(t)\right) & f_{y 2}\left(y_{2}(t)\right)
\end{array}\right] \\
& +\left[\begin{array}{c}
0 \\
4476+f_{u 2}\left(y_{2}(t)\right)
\end{array}\right] u(t)
\end{aligned}
$$

The unknown functions $f_{y 1}\left(y_{1}\right)$ and $f_{u 2}\left(y_{1}\right)$ are used to encompass nonlinearities due to piezoelectric hysteresis and nonlinear actuator gain as reflected in system gain and natural frequency behavior, and unknown function $f_{y 2}\left(y_{2}\right)$ accounts for uncertainty about the damping coefficient of the system. These nonlinear functions are estimated to have bounds of

$$
\begin{aligned}
& \left|f_{y 1}\left(y_{1}(t)\right)\right| \leq 1839\left|y_{1}(t)\right|, \\
& \left|f_{y 2}\left(y_{2}(t)\right)\right| \leq 2.4\left|y_{2}(t)\right|, \\
& \left|f_{u 2}\left(y_{2}(t)\right)\right| \leq 223\left|y_{2}(t)\right| \\
& \left|f_{y 1}\left(y_{1}^{\prime}(t)\right)-f_{y 1}\left(y_{1}(t)\right)\right| \leq 7718\left|y_{1}^{\prime}(t)-y_{1}(t)\right| \\
& \left|f_{y 2}\left(y_{2}^{\prime}(t)\right)-f_{y 2}\left(y_{2}(t)\right)\right| \leq 9.6\left|y_{2}^{\prime}(t)-y_{2}(t)\right| \\
& \left|f_{u 2}\left(y_{2}^{\prime}(t)\right)-f_{u 2}\left(y_{2}(t)\right)\right| \leq 446\left|y_{2}^{\prime}(t)-y_{2}(t)\right|
\end{aligned}
$$

based on prior experimentation with thin-film piezoelectric actuators. These bounds reflect approximately 5\% uncertainty in spring stiffness and gain and 20\% uncertainty in damping ratio. These levels are actually much larger than uncertainty about the meso-scale piezoelectric actuator used for experimental testing [1] but consistent with model uncertainty for micro-scale piezoelectric actuators [15] for which the control algorithm is ultimately targeted (micro-scale actuators are

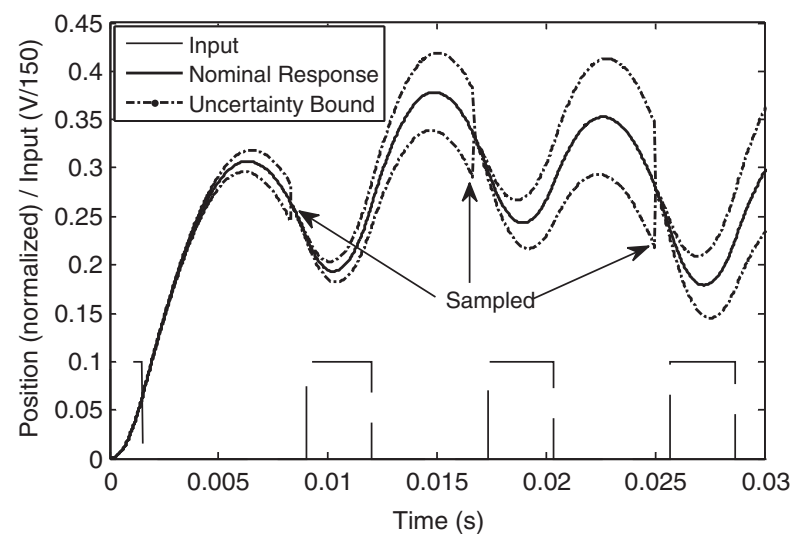

Fig. 4. Sample Nominal ResPonse to a Switching Input and bounds on uncertainty in output relative to response due to Bounded, Unknown nonlinearities.

not yet used for experimental testing as current prototypes lack integrated sensing, although such sensors have been demonstrated on vertical piezoelectric actuators created by the same microfabrication process).

Under the uncertainty listed, matrices $\boldsymbol{C}_{y}, \boldsymbol{C}_{u}, \boldsymbol{C}_{y}^{\prime}$, $\boldsymbol{C}_{u}^{\prime}$ become

$$
\begin{array}{ll}
\boldsymbol{C}_{y}=\left[\begin{array}{cc}
0 & 0 \\
1839 & 2.4
\end{array}\right], \quad \boldsymbol{C}_{u}=\left[\begin{array}{cc}
0 & 0 \\
223 & 0
\end{array}\right], \\
\boldsymbol{C}_{y}^{\prime}=\left[\begin{array}{cc}
0 & 0 \\
7718 & 9.6
\end{array}\right], & \boldsymbol{C}_{u}^{\prime}=\left[\begin{array}{cc}
0 & 0 \\
446 & 0
\end{array}\right]
\end{array}
$$

The 'on' input for the system tested is taken to be $u_{\max }=$ $15 \mathrm{~V}$, and samples are taken every $0.083 \mathrm{~s}$.

Using the system model above, $x^{K}$ and $\Delta^{K}$ are first calculated numerically for ranges of potential switching times, $0<\tau_{p}<0.0083 \mathrm{~s}, 0.0083 \mathrm{~s}<\tau_{s}<0.0166 \mathrm{~s}$, and $0<\tau_{d}<0.0083 \mathrm{~s}$. For an illustration of the uncertainty margins in comparison to the nominal response, Fig. 4 shows a sample response when $\tau_{p}=0.001 \mathrm{~s}$, $\tau_{s}=0.009 \mathrm{~s}$, and $\tau_{d}=0.003 \mathrm{~s}$ surrounded by the associated error bounds, with sensor updates to the estimated output at the sampling points. As can be seen, error bounds grow over time, such that there use to draw conclusions about the system is limited to transient responses, with typical duration on the order just a few times the period of an underdamped system such as that in this example.

Next, the nominal response in the next iteration, $\boldsymbol{x}^{K+1}$, uncertainty in the full response, $\boldsymbol{\Delta}^{K+1}$, and uncertainty in the change in response with respect to switching times, $\Delta_{D T}^{K+1}$ and $\Delta_{I C}^{K+1}$ are calculated numerically for hypothetical changes in switching 
times $\delta \tau_{p}^{K+1}, \delta \tau_{s}^{K+1}$, and $\delta \tau_{d}^{K+1}$. As an illustration of the behavior observed, Fig. 5(a) shows the change in nominal response at the first measurement point, $x_{p}^{K+1}-x_{p}^{K}$ as a function of $\tau_{p}^{K}$ and $\delta \tau_{p}^{K+1}$, while Fig. 5(b) shows the total uncertainty at time $t_{1}$, $\Delta_{D T}^{K+1}\left(t_{1}\right)+\Delta_{I C}^{K+1}\left(t_{1}\right)$. For this first output, we observe that the effect of a change in switching time $\tau_{p}$ has a much larger effect on the output than uncertainty at time $t_{1}$, so that increasing $\tau_{p}$ can be reliably expected to increase $y_{p}$ for this systems. The latter outputs, $y_{s}$, and $y_{d}$, face much greater uncertainty in the response, due to the growing error bounds, and more complicated dependence on switching times, with limits on $\tau_{s}^{K}$ and $\delta \tau_{s}^{K+1}$ being especially strict. Fig. 6 shows the maximum change, $\delta \tau_{s}^{K+1}$, for various values of $\tau_{s}^{K}$ and $\tau_{d}^{K}$ that satisfies the desired properties for $x_{s}$ and $x_{d}$ from (33), for a sample value of $\tau_{p}, \tau_{p}=0.003 \mathrm{~s}$.

Tabulating all $\tau_{p}^{K}, \tau_{s}^{K}$, and $\tau_{d}^{K}$ for which (14) is satisfied for $\delta \tau_{p}^{K+1}<100 \mu \mathrm{s}, \delta \tau_{s}^{K+1}<10 \mu \mathrm{s}$, and $\delta \tau_{d}^{K+1}<100 \mu$ s produces ranges $\tau_{p}^{*}, \tau_{s}^{*}$ and $\tau_{d}^{*}$ as shown in Fig. 7(a). To convert these bounds on changes in switching times to adaptation law gains, the maximum and minimum possible values for each output are calculated, to produce respond to a perturbation in switching by pushing the response out of the set discarded. The outcome of the analysis, then, is that for various references, $r$, a set of $\tau_{p}^{* c}, \tau_{s}^{* c}$ and $\tau_{d}^{* c}$ may potentially be found that can be used to select an initial switching time guess, from which the adaptation algorithm will converge to the corresponding target values.

\section{SIMULATION AND EXPERIMENTAL RESULTS}

Controller performance was first tested in simulation on a variety of linear and nonlinear systems. A sample response is shown in Fig. 8, for the following nonlinear system having the same nominal linear dynamics as the actuator used for experimental testing,

$$
\begin{aligned}
\dot{y}_{1}(t)= & y_{2}(t) \\
\dot{y}_{2}(t)= & -38400 y_{1}(t)-1920 y_{1}(t) \sin \left(10 y_{1}(t)\right) \\
& -12 y_{2}(t)-2.4 y_{2}(t)+32300 u(t)
\end{aligned}
$$

$$
\begin{aligned}
& \gamma_{p}<\frac{\delta \tau_{p, \max }}{\max _{\substack{\tau_{p}, \tau_{s}, \tau_{d} \in \tau_{p}^{*}, \tau_{s}^{*}, \tau_{d}^{*} \\
\delta \tau_{p}<\delta \tau_{p, \max }}}\left(\left|x_{p}^{k+1}-x_{p}^{k}\right|+\Delta^{k+1}\left(t_{1}\right)\right)} \\
& \gamma_{s}<\frac{\delta \tau_{s, \max }}{\max _{\substack{\tau_{p}, \tau_{s}, \tau_{d} \in \tau_{p}^{*}, \tau_{s}^{*}, \tau_{d}^{*} \\
\delta \tau_{s}<\delta \tau_{s, \max }}}\left(\left|x_{s}^{k+1}-x_{p}^{k+1}-x_{s}^{k}-x_{p}^{k}\right|+\Delta^{k+1}\left(t_{2}\right)+\Delta^{k+1}\left(t_{1}\right)\right)} \\
& \gamma_{d}<\frac{\delta \tau_{d, \max }}{\max _{\substack{\tau_{p}, \tau_{s}, \tau_{d} \in \tau_{p}^{*}, \tau_{s}^{*}, \tau_{d}^{*} \\
\delta \tau_{d}<\delta \tau_{d, \max }}}\left(\left|x_{d}^{k+1}-x_{d}^{k}\right|+\frac{1}{Q-1} \sum_{q=1}^{Q} \Delta^{k+1}\left(t_{q}\right)\right)}
\end{aligned}
$$

giving feasible $\gamma_{p}<0.011, \gamma_{s}<0.0091$, and $\gamma_{d}<0.0085$ for this specific system (note that $K$ and $K+1$ do not correspond to a specific time step above, but are retained in (40) to signify the change in response for each candidate switching time and increment in switching time evaluated). In addition, candidate reference values, $r$, for convergence analysis are selected using (35), with $0.16<r<0.65$ guaranteed for some values of $\tau_{p}^{K}, \tau_{s}^{K}$, and $\tau_{d}^{K}$ in $\tau_{p}^{*}, \tau_{s}^{*}$, and $\tau_{d}^{*}$. The closed set of switching times satisfying (34) as well as (14) for a sample target of $r=0.5$ and $\delta \tau_{p}^{K+1}<100 \mathrm{~ms}, \delta \tau_{s}^{K+1}<10 \mathrm{~ms}$, and $\delta \tau_{d}^{K+1}<100 \mathrm{~ms}$ is indicated by darker dots in Fig. 7, with candidate switching times from the margins of the tested region and those that could, given uncertainty,
Convergence was evaluated for a step duration of $0.025 \mathrm{~s}$ as above, using $\gamma_{p}=0.01, \gamma_{s}=0.002$, and $\gamma_{d}=0.008$ for the adaptation parameters and $u_{\max }=$ 15. We observe generally fast adaptation of the simulated systems to which the controller is applied, with convergence typically occurring in fewer than 30 time steps. Experience with simulations indicated, as would be expected, that the convergence analysis is conservative, as systems are frequently observed to converge to target outputs when given initial switching time guesses or adaptation gains outside the guaranteed ranges for convergence. On the other hand, pure guessing of adaptation gain values rarely results in effective convergence, such that the convergence analysis was quite useful in controller testing on simulated systems. 

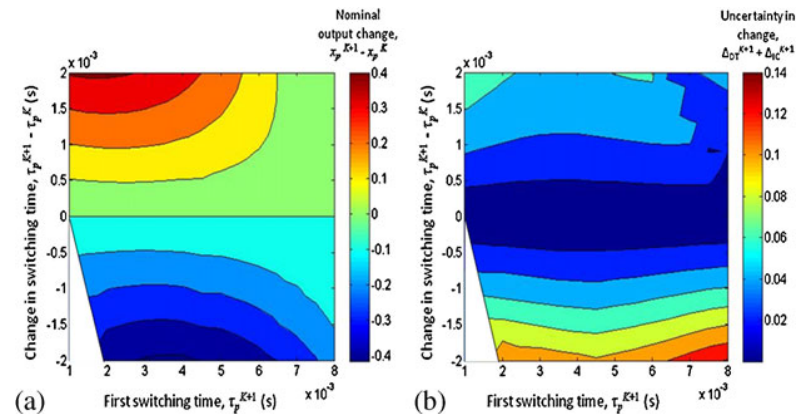

Fig. 5. (a) Nominal Change In Output at First Measurement Point $\left(x_{p}\right)$ As a function of First switching time in current iteration $\left(\tau_{p}\right)$ and change in First switching time Next Iteration $\left(\delta \tau_{p}\right)$ and (b) Magnitude of possible error in estimate of Change in output at first measurement $\operatorname{oint}\left(\left|y_{p}^{K+1}-x_{p}^{K+1}-y_{p}^{K}-x_{p}^{K}\right|\right)$.

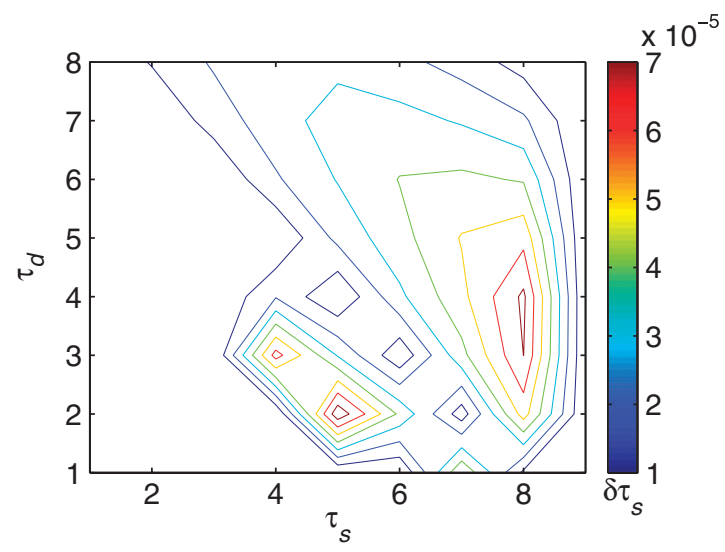

Fig. 6. Maximum change in second switching time $\left(\delta \tau_{S}\right)$ satisfying convergence Criteria As a function of switching times $\tau_{s}$ and $\tau_{d}$ for $\tau_{p}=0.003 \mathrm{~s}$.

To evaluate the performance of the proposed iterative on-off controller experimentally, the controller is applied to a piezoelectric actuator test bed shown in Fig. 9. The actuator tested was a $40 \mathrm{~mm}$ long, $10 \mathrm{~mm}$ wide Ceratec bimorph actuator with a natural frequency of approximately $100 \mathrm{~Hz}$. A strain gage was attached to the actuator to measure deflection, with output of the system being output voltage of the strain gage sensing circuit normalized to an output of 1 when the actuator is at its maximum steady-state displacement. Mass was added to the tip of the actuator to reduce the natural frequency of the experimental apparatus to $31.26 \mathrm{~Hz}$ and the damping ratio of the experimental system was 0.0307 , to resemble the properties of a projected microrobotic leg carrying a payload.

The control signal is generated on a TMS320F28 335 digital signal processor, with an H-bridge circuit

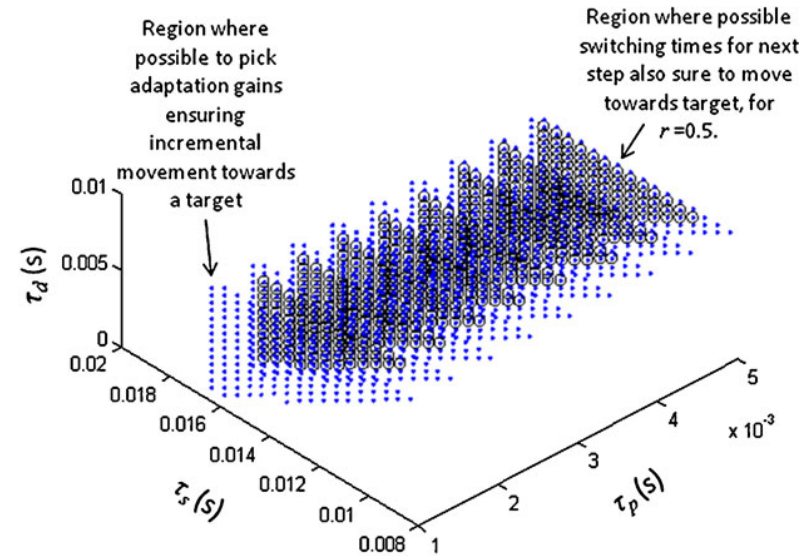

Fig. 7. Region of switching times for which some incremental change in switching time satisfies convergence criterion for at least one iteration (dotted points); closed region of switching times satisfying convergence conditions when $R=0.5$ (circled points).

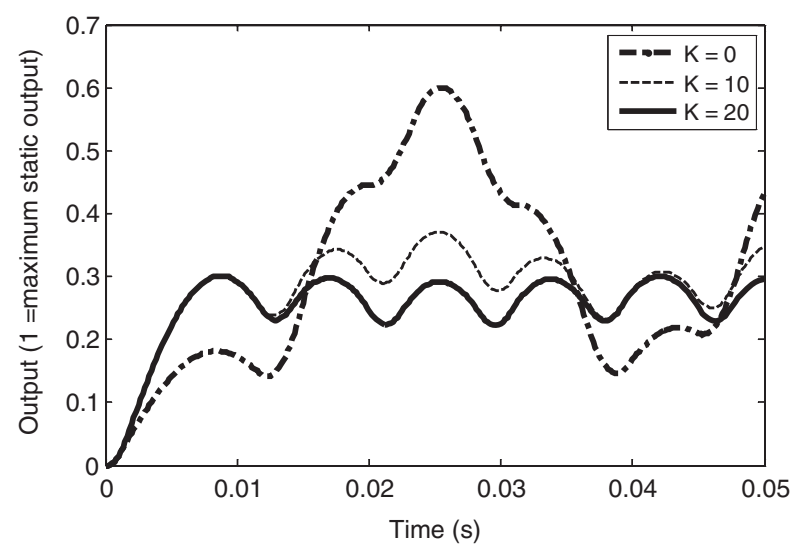

Fig. 8. Simulation response of an arbitrary nonlinear function satisfying nonlinearity bounds.

acting as the on-off interface between the low-voltage DSP and a $15 \mathrm{~V}$ supply for the actuator. For testing a converging case, the controller used $\gamma_{p}=0.009$, $\gamma_{s}=0.009, \gamma_{d}=0.0002, f=150 \mathrm{~Hz}$, and measurements taken every $0.0083 \mathrm{~s}$, or at a $120 \mathrm{~Hz}$ sampling rate. For testing a non-converging case, the controller used $\gamma_{p}=0.024, \gamma_{s}=0.009, \gamma_{d}=0.0002, f=150 \mathrm{~Hz}$, and $t_{s}=0.0083 \mathrm{sec}$. As during the convergence analysis process of the simulated system (37), these control constants, driving frequency, and sample time are chosen to converge or to non-converge to a target reference level at a final adaptation cycle.

Sample responses for two scenarios are shown in Fig. 10(a) and (b). The experimental result with converging parameters show a successful convergence 


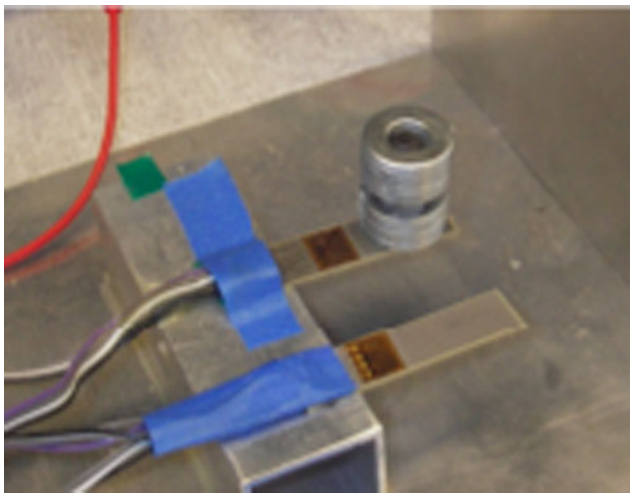

Fig. 9. A piezoelectric actuator testbed.
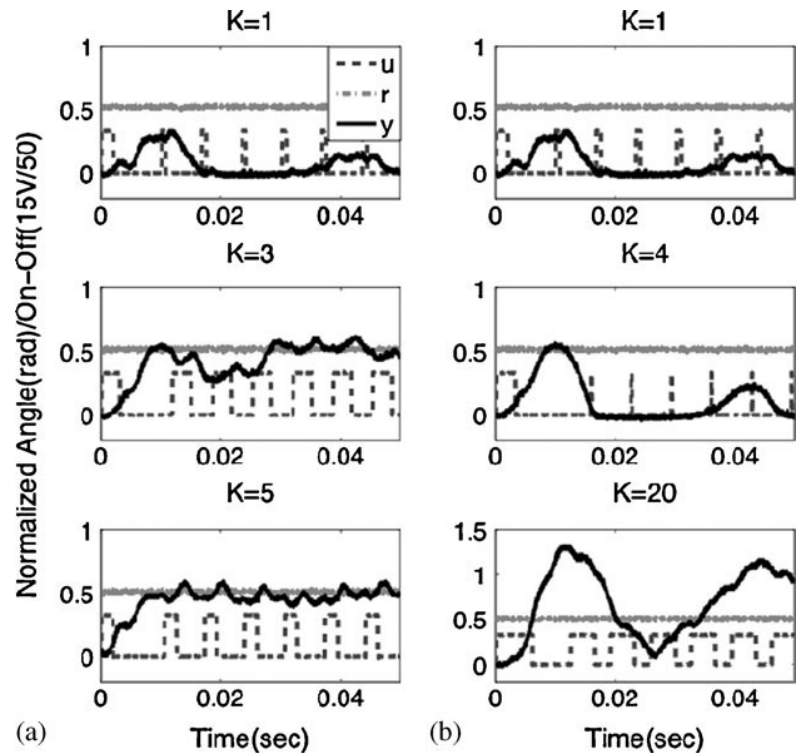

Fig. 10. Sample experimental response: (a) converging case and (b) non-converging case.

to the target reference level and has similar convergence trend to the simulation result. In the final adaptation cycle, system output rises successfully to the target reference level with minimal overshoot, and holds the system at the reference level using a limited number of on-off switching transitions. In addition, these results satisfy well the convergence criteria (14) and (15) as shown in Table I. Once converged, the system maintains its behavior over many additional iterations, with up to several minutes repeated, with testing limited by data collection limits. However, there exists higher-order mode oscillation visible during the transient phase, possibly due weakness of the connection with the added mass. On the contrary, the experimental result with non-converging parameters does not show a successful convergence to the target reference level even if the adaptation cycle is increased.
Table I. Measurement errors as K goes to infinity at Figure 10(a).

\begin{tabular}{lccc}
\hline & $\left|y_{p}^{K}-r\right|$ & $\left|y_{s}^{K}-y_{p}^{K}\right|$ & $\left|y_{d}^{K}-r\right|$ \\
\hline$K=1$ & 0.268 & 0.088 & 0.468 \\
$K=3$ & 0.052 & 0.064 & 0.012 \\
$K=5$ & 0.012 & 0.008 & 0.008 \\
\hline
\end{tabular}

\section{DISCUSSION}

The primary benefit to implementing a servo controller in this manner is that it requires much less power than PWM or analog controllers implemented on piezoelectric actuators. This will be extremely useful for autonomous micro-robots based on thin-film leadzirconate-titanate (PZT) microactuators. For a typical microactuator capacitance of $1 \mathrm{nF}$ at $20 \mathrm{~V}$, energy consumption to charge the actuator with a transistor switching circuit is approximately $0.6 \mu \mathrm{J}$ per switch, when both capacitive loads and leakage currents are taken into effect, as measured on the prototype leg joint. Total power consumption in actuation and drive circuitry of the proposed control system, as well as PWM control schemes, then depends on the switching frequency utilized. Meanwhile, power consumption for position sensing likewise increases with frequency. As an illustration, current trends in low-power sensing circuitry for capacitive sensors, a sensing modality commonly utilized in MEMS, reflect an approximate exponential relationship between power and sampling frequency, in $\mu \mathrm{W}$, of $1.6 f^{0.65}$, derived by compiling power consumption data from recent low-power capacitive sensing circuits $[16,17]$.

Given the state-of-the art circuit capabilities above, it is possible to illustrate power consumption versus the effective driving frequency and sampling frequency of piezoelectric actuator control, as shown in Fig. 11. Total power consumed is taken to be power required to charge a sample piezoelectric actuator at various driving frequencies plus the power consumption of capacitive sensor operating at various sampling frequencies. The regions showing at least local convergence for the prototype micro-robot leg examined in this paper using on-off control and a common PWM algorithm are marked. The PWM algorithm shown for comparison is a feedback PID controller designed to bring the nominal linear system to the reference target with zero overshoot and comparable rise time to the on-off controller; the analog output of the PID controller is applied to the piezoelectric actuation via pulse-width modulation at the driving frequency. As can be seen, limiting the number of switching transitions per leg 


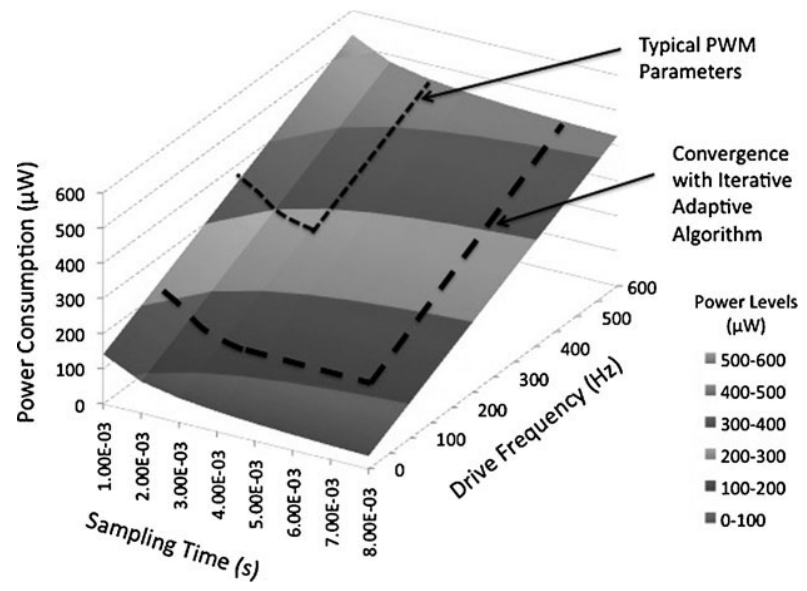

Fig. 11. Power Consumption versus sampling time and driving frequency for a $1 \mathrm{nF}$ Capacitive microactuator with nominal natural frequency of $43 \mathrm{~Hz}$, Damping Ratio of $4 \%$, and 'On' voltage of $20 \mathrm{~V}$.

movement (effectively drive frequency) and number of measurements needed per motion (effectively sensing frequency) can have a major effect on total system power consumption.

The controller proposed here does face significant limitations, however, due to its specialization for simple implementation and power savings. While convergence analysis can be applied to an arbitrary system, it is complicated, and the numerical integrations involved require significant calculation time. The corresponding benefit of the approach is that convergence analysis may be performed entirely off-line, providing guarantees on convergence when only running the very simple adaptation algorithm within an autonomous system. In all cases, some bounds on nonlinearities of the system must be known, and convergence analysis will not generally work for long time durations of motion, due to growing uncertainty about the response. The controller as utilized on piezoelectric actuators here is thus specifically intended to adapt transient motions. Furthermore, convergence guarantees are only local and are conservative, with a definite region of convergence obtained but unlikely to show all initial conditions and target motions that in reality would converge. And, fundamentally, the control approach is only applicable to systems where the same motion is to be repeated many times, with approximately 10 iterations typically needed, and as many as 40 required in some cases, to converge to the desired step motion in simulation and experimental testing. As a practical matter, implementation by the controller designer requires selection of a desired driving frequency for the steady-state portion of motion that balances power usage and oscillation between switching instants for a given system.

Nonetheless, for autonomous micro-robotics this controller has the potential to be a powerful tool in regulation of walking gaits. Changing environments and terrains can cause significant changes in dynamics, yet model-based identification is comparatively power and computation intensive. On the other hand, the small size of micro-robots would typically correspond to hundreds of repeated steps in any given condition, while the proposed algorithm utilizes relatively simple calculations and low sampling within the robot processor to bring about efficient motion. The smooth step output, for the desired output measurements selected, is useful for quasi-static walking, in which a partial set of legs drive the robot forward and hold it in place while lowering the remaining legs, which then begin another step. Finally, our experiments in simulation and on macro-scale piezoelectric actuator testbeds indicate that while the range of error in initial switching time estimates may not be determined from our existing convergence test, the controller tolerates large amounts of error in initial guesses, so long as the other convergence checks on the nominal system (such as adaptation parameters and driving and switching frequencies) are satisfied. In addition, optimization algorithms for nominal system models, while overly complex for on-board processing, are available, and may be used to assure that initial estimates for on-off switching times are near their desired targets [18].

While the specific controller explored in the bulk of the paper relies on three specific switching time parameters and three specific functions of the measurements, the iterative adaptation of switching times can be generalized to follow other transient responses using the switching time definitions in (4) and the comparison of nominal responses and their uncertainty from Section 4.2. For the general case, convergence is most easily analyzed when adapting a single switching time between each measurement. In this scenario, convergence can be analyzed sequentially, working forward from the target output at the first measurement. If so, the challenge becomes identifying target points that are guaranteed to be reachable for each measurement time. While growing uncertainty eventually will prevent conclusions from being drawn about whether convergence in the next measurement point can be guaranteed, a novelty of this approach is that sensor measurements can be planned for any point in time, and not at a fixed rate, unlike most control systems. In addition, it should be noted that full state feedback at any point in time allows uncertainty in the ensuing response to be 'reset' 
such that convergence analysis can be continued further along the transient response.

\section{CONCLUSION AND FUTURE WORK}

A model-free, iterative adaptive controller for on-off control of dynamic systems, is proposed and associated behavior with respect to control of piezoelectric microactuators for autonomous micro-robots is explored. The controller enables very low-power control of such actuators when many stepping motions are to be regulated. With careful selection of objectives by the designer, convergence can be obtained with relatively few output measurements, and on-off control lends itself to implementation with very little power and simple sensors and actuators. Simulation studies indicate a wide range of controller applicability for individual selections of controller parameters, particularly in the range needed for control of prototype micro-robot leg joints. In experimental testing on a macro-scale testbed, we have observed good convergence in the presence of modest nonlinearities and noise. Future work will seek more general statements on rate of convergence, as well as means of extending the duration of transient motions that can be accommodated by the controller.

\section{REFERENCES}

1. Edamana, B., B. Hahn, J. Pulskamp, R. Polcawich, and K. Oldham, "Modeling and optimal low-power on-off control of thin-film piezoelectric rotational actuators," IEEE/ASME Trans. Mechatron., PP(99), pp. 1-13 (2010).

2. Oldham, K., B. Hahn, B. Edamana, J. Pulskamp, and R. Polcawich, "Low-power switching control schemes for micro-robotic actuators," Proc. ASME Smart Mater. Adapt. Struct. Intell. Syst. Conf., Ellicot City, MD (2008).

3. Main, J. A., D. Newton, L. Massengill, and E. Garcia, "Efficient power amplifiers for piezoelectric applications," Smart Mater. Struct., Vol. 5, pp. 766-775 (1996).

4. Kaya, C. Y. and J. L. Noakes, "Computational method for time-optimal switching control," $J$. Optim. Theory Appl., Vol. 117, No. 1, pp. 69-92 (2003).

5. Singhose, W. E., B. W. Mills, and W. P. Seering, "Closed-form methods for on-off control of multimode flexible structures," Proc. 36th Conf. Decis. Control, San Diego, CA, pp. 1381-1386 (1997).
6. Chen, C.-T. and C. Hwang, "Optimal on-off control for fed-batch fermentation processes," Ind. Eng. Chem. Res., Vol. 29, pp. 1869-1875 (1990).

7. Oldham, K., B. Hahn, and P. Park, "On-off control for low-power servo control in piezoelectric microrobotics," Proc. 2008 ASME Dyn. Syst. Control Conf., Ann Arbor, MI (2008).

8. Breddermann, R., "Realization and application of a self-tuning on-off controller," Lecture Notes in Control and Information Sciences, Springer, Berlin, Germany, pp. 74-83 (1980).

9. Golob, M., B. Tovornik, and D. Donlagic, "Comparison of the self-tuning on-off controller with the conventional switching controllers," $1 s t$ IEEE Conf. Control Appl., 2, Dayton, OH, pp. 962963 (1992).

10. Lim, C. W., T. Y. Chung, and S. J. Moon, "Adaptive bang-bang control for the vibration control of structures under earthquakes," Earthq. Eng. Struct. Dyn., Vol. 32, pp. 1977-1994 (2003).

11. Ahn, K. and S. Yokota, "Intelligent switching control of pneumatic actuator using on/off solenoid valves," Mechatronics, Vol. 15, pp. 683-702 (2005).

12. Zhang, J., N. Wang, and S. Wang, "Fussy-neuron intelligent coordination control for a unit power plant," Asian J. Control, Vol. 3, No. 1, pp. 57-63 (2001).

13. Spall, J. and J. A. Christion, "Model-free control of nonlinear stochastic systems with discrete-time measurements," IEEE Trans. Autom. Control, Vol. 43, No. 9, pp. 1198-1210 (1998).

14. Hou, Z. and W. Huang, "The model-free learning adaptive control of a class of SISO nonlinear systems," Proc. Am. Control Conf., Albequerque, NM, pp. 343-344 (1997).

15. Hahn, B. and K. Oldham, "Model-free adaptive on-off step controller for piezoelectric microrobotics," Proc. 2009 ASME Dyn. Syst. Control Conf., Hollywood, CA (2009).

16. Fang, D., H.-W. Qu, and H.-K. Xie, "A $1 \mathrm{~mW}$ dual-chopper amplifier for a $50-\mu \mathrm{g} / \sqrt{ } \mathrm{Hz}$ CMOSMEMS accelerometer," 2006 Symp. VLSI Circuits, Honolulu, HI (2006).

17. Bracke, W., P. Merken, R. Puers, and C. Van Hoof, "Ultra-low-power interface chip for autonomous capacitive sensor systems," Circuits Syst. I, Vol. 54, No. 1, pp. 130-140 (2007).

18. Oldham, K. and B. Edamana, "An optimal onoff controller with switching costs using non-linar binary programming," Proc. 2009 Am. Control Conf., St. Louis, MO (2009). 


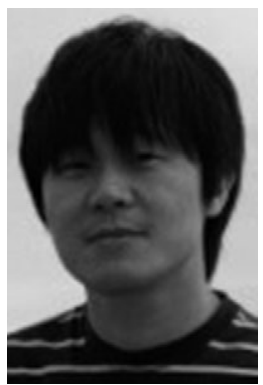

Bongsu Hahn received his B.S. degree in Mechanical Engineering from Yeungnam University, Gyeongsan, Korea, in 1999, and his M.S. degree in Mechatronics Engineering from Gwangju Institute of Science and Technology, Gwangju, Korea, in 2002. From 2002 to 2007, he was a Researcher with the Actuation and Control Team for Missile Guidance, Agency for Defense Development, Korea. Since 2007, he has been with the Vibration and Acoustic Laboratory: Microsystems, University of Michigan, AnnArbor. His current research interests include low power switching control for microdevices, coordinating highly efficient voltage conversion, and adaptive control.

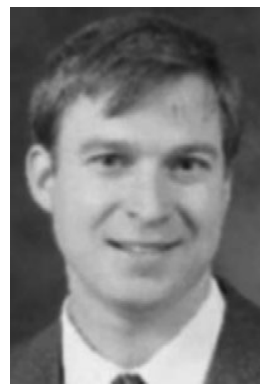

Kenn Oldham received his B.S. degree in Mechanical Engineering from Carnegie Mellon University, Pittsburgh, PA, in 2000 and his Ph.D. degree in Mechanical Engineering from the University of California, Berkeley, in 2006. He is currently an Assistant Professor in the Department of Mechanical Engineering, University of Michigan, Ann Arbor, MI. His research interests include microactuator design and applications, optimal design and control, design for controllability, and efficient sensing and power strategies for microelectromechanical system devices. Prof. Oldham is a member of the American Society of Mechanical Engineers. 